\title{
INFLUENCE OF COMBINE APPLICATION OF TOPIC, GRANSTAR HERBICIDES AND PHOSPHOR LEVELS ON WEEDS AND BREAD WHEAT YIELD AND ITS COMPONENTS
}

\author{
Mohammed Ali Hussain ${ }^{*}$, Abbas Alo Khether ${ }^{1}$ and Mohammed Othman Mohammed ${ }^{2}$ \\ ${ }^{1}$ Dept. of Field Crop, College of Agriculture, University of Dohuk, Kordistan Region- Iraq \\ ${ }^{2}$ Dept. of Biology, College of Sciences, University of Zakho, Kordistan Region- Iraq
}

(Received: March 12, 2018; Accepted for publication: August, 13, 2018)

\begin{abstract}
A field trail was conducted at the researches Farm of Agricultural College of Duhok University, Kurdistan Region/ Iraq, which located in semi-arid zone during the season 2016-2017 in two planting dates (28 November and 28 December 2016 ) to study the influence of four integrate Topic and granstar $(0,1200$, $1600,2000 \mathrm{ml}^{\circ} \mathrm{ha}^{-1}$ and $\left.0,20,40,60 \mathrm{gm} \mathrm{ha}^{-1}\right)$ and four phosphor fertilizer levels at $\left(0,183,229,275 \mathrm{~kg} \mathrm{ha}^{-1}\right)$ respectively, on weeds and bread wheat yield and its components. The experiment unit arranged in factorial experiment, using Randomize Complete Block Design (RCRD) with three replications. The results showed that herbicide and phosphorous levels exhibited significant effect on weeds and yield and its components. The treatment (Topic $2000 \mathrm{ml}+60 \mathrm{~g} \mathrm{ha}^{-1}$ granstar) produced maximum reduction in total weeds growth and recorded maximum values for $51.1 \mathrm{~g}$ ) respectively. High dose of herbicide (Topic:2000 ml. $\mathrm{ha}^{-1}$ and Granstar, $60 \mathrm{~g} \mathrm{ha}^{-1}$ ) and high of of grains spike $\mathrm{e}^{-1}, 1000$-grain weight and grain yield which were $(45.4,42.5 \mathrm{~g}$, and high phosphorus levels $\left(275 \mathrm{~kg} \mathrm{ha}^{-1}\right)$ are recommended for weed control and high yield and a good quality wheat grains.
\end{abstract}

KEYWORD: Herbicide, weeds, Phosphorus, wheat, yield

\section{INTRODUCTION}

W heat (Triticum aestivum $\mathrm{L}$ ) is one of the most important crop in the world and the widest distribution among cereal crops. It is an important cereal crop in Iraqi Kurdistan region. Its average productively is low comparing to other advance countries in the world. Several practices such as weed control, and adequate fertilizers are very important for production of bread wheat (23). The weeds compete with crop plants for essential growth factors like light, moisture, nutrients and space (8). Weed can also increase harvesting cost, reduce quality of product. Thus found that uncontrolled weed growth throughout the crop growth a $57.6 \%$ to $73.2 \%$ yield reduction, while , (22) reported the yield losses due to weed are 17 to $25 \%$. The chemical weed control is more economical than conventional method (9). Several researchers (18), (15), and (3) . Consider that the chemical method of weeds control proved to be more efficient method in controlling weeds. (19), showed that phosphorus revealed as the second most important factor, and essential for plant growth after nitrogen. While, Mata and Brown showed the phosphor in soil was high fixation and low levels of avarable $p$, the applications of $p$ in bands generally increase productivity, (13) exhibited that phosphorus is taken up by plants as an orthophosphate ions $\left(\mathrm{H}_{2} \mathrm{Po}_{4}{ }^{-1}\right.$ and $\left.\mathrm{HPO}_{4}^{-2}\right)$ which play a vital function to maintain yield and quality. When application of phosphorus in adequate quantity and available source to plant medium has a vital role for plant growth, yield production, and improving yield quantity of wheat, also phosphorus is essential for cell division seed and fruit development (1). Several researchers (6), (5), (4) and (7), were studied different soil and found that different parameters affected the availability of soil phosphorus to plant such as $\mathrm{PH}, \mathrm{Ca}^{+2}$ Concentration, moisture, texture , root density, $\mathrm{CaCo}_{3}$ amount of organic matter content and type of clay mineral, also obtained, the maximum phosphorus doses contributed in achieving maximum 1000-grain weight, dry matter and highest number of grain spike ${ }^{-1}$. The present study was devoted to study the yield and yield components of wheat as affected by herbicide and phosphorus fertilizer application and weed growth and population. 


\section{MATERIAL AND METHOD}

This trail was conducted at the farm of Agriculture collage, University of Duhok, Iraqi Kurdistan Region, which located in semi-arid zone during the 2016-2017 season in two planting dates under rain feed conditions to estimate the influence of four integrate Topic and granstar herbicides $\left(1200,1600,2000 \mathrm{ml}^{-\mathrm{ha}^{-1}}\right.$ and , 20, 40, $\left.60 \mathrm{gm} \mathrm{ha}^{-1}\right)$ and four phosphorus levels. (0, 183, $229,275 \mathrm{~kg} \mathrm{ha}^{-1}$ ) on weeds and wheat yield and it's components. The experimental units were arranged in factorial experiment, using Complete Randomize Block Design and replicated three times. All physiochemical properties of top soil sample (0-30) and weather properties shown in (Table 1). The experimental units consists from four rows with three meter length and $20 \mathrm{~cm}$ distance between rows. Nitrogen was added at rate $120 \mathrm{~kg} \mathrm{ha}^{-1}$ in form of urea $46 \% \mathrm{~N}$. Half dose of nitrogen and all doses of phosphorus added before planting date, the second half of nitrogen added at tillering stage. The seeds were planted in 28 November and 28 December 2016 with seeding rate $120 \mathrm{k} \mathrm{ha}^{-1}$ using TAWAHI-13 bread wheat (Triticum aestivum) variety The date of spraying the integrate herbicide were 7 and 15 July 2017 when the plat 2-3 leave stage. Weed sampling recorded after 100 days from planting date. The dry weight of broad and narrow leaved weeds of $1 \mathrm{~m}^{2}$ matured from each plot, were and dried using over drying at $\left(75 \mathrm{c}^{0}\right)$ for (48) hrs, (25).

Data collected on ten plants selected randomly from four rows, included a characters of broad and narrow leaved weeds in one $\mathrm{m}^{2}$, plant height, leaf area $\mathrm{cm}^{2}$,days to flowering, number of grains spike $^{-1}$, grains weight spike ${ }^{-1}, 1000$ - grain weight and grain yield. The data analyzed using Minitab software. Duncan Multiple Range Test (DMRT) was used to test the means of treatments.

Table (1): Physiochemical properties of top soil sample (0-30) and weather properties.

\begin{tabular}{|c|c|c|c|c|c|c|}
\hline \multicolumn{5}{|c|}{ Weather properties } & \multicolumn{2}{|l|}{ soil properties } \\
\hline Month & $\begin{array}{l}\text { Ave. Daily } \\
\text { max.tem c }\end{array}$ & $\begin{array}{l}\text { Ave. Daily } \\
\text { min..tem } c^{0}\end{array}$ & $\begin{array}{c}\text { Seasonal } \\
\text { Relative } \\
\text { Humidity RH \% }\end{array}$ & $\begin{array}{c}\text { Seasonal } \\
\text { Rainful mm }\end{array}$ & & \\
\hline \multicolumn{5}{|c|}{2016} & \multirow[t]{2}{*}{ Sand $\left(\mathrm{g} \cdot \mathrm{kg}^{-1}\right)$} & \multirow[t]{2}{*}{89.60} \\
\hline October & 36.2 & 18.3 & 32.3 & 00 & & \\
\hline November & 12.1 & 3.6 & 40.9 & 10.2 & Silt $\left(g \cdot \mathrm{kg}^{-1}\right)$ & 486.90 \\
\hline December & 11.6 & 2.04 & 79.4 & 72 & Clay $\left(\mathrm{g} \cdot \mathrm{kg}^{-1}\right)$ & 419.85 \\
\hline \multicolumn{5}{|c|}{2017} & Soil texture & Silty clay \\
\hline January & 12.23 & -0.56 & 73.47 & 29.1 & $\mathrm{PH}$ in soil past & 8.25 \\
\hline February & 13.76 & -57.2 & 65.8 & 17.6 & Ec $\left(\mathrm{dsm}^{-1}\right)$ at $25 \mathrm{c}^{0}$ in soil past & 0.55 \\
\hline March & 18.53 & 6.881 & 67.05 & 52.2 & $\mathrm{CaCo} 3\left(\mathrm{~g} \mathrm{~kg}^{-1}\right)$ & 205.2 \\
\hline April & 24.21 & 9.52 & 63.98 & 81.4 & Organic matter $\mathrm{k} \mathrm{ha}^{-1}$ & 145 \\
\hline May & 24.4 & 37.8 & 19 & 15.0 & Available $\mathrm{N} \mathrm{k} \mathrm{ha}^{-1}$ & 0.22 \\
\hline Total & & & & 277.5 & & \\
\hline
\end{tabular}

* Ec: Electric conductivity

\section{RESULTS AND DISCUSSIONS}

Statistical analysis of data that showed all herbicidal treatments had significant effect on all studied traits in both seeding date with the exception to plant height and leaf area in the second date. The phosphorus levels exhibited considerable effects in all traits in first and second date with the exception of number and dry broad leaved weeds, plant height, leaf area and grain weight spike ${ }^{-1}$. The interaction between herbicides and phosphorous levels gave significant and significant effects on all traits except of dry weight of broad leaved weeds, plant height, leaf area and grain weight spike ${ }^{-1}$ in the first date and plant height, leaf area and number of grains spike ${ }^{-1}$ in second date.

Similar impact of herbicides and phosphorus levels on 1000-grain, number of grains spike ${ }^{-1}$ and grain yield were observed (12) and (10). 
Table (2): Mean square analysis of integrate herbicide and phosphorus levels on broad and narrow leaf weeds. components in first and second planting date.

\begin{tabular}{|c|c|c|c|c|c|c|c|c|c|c|c|c|}
\hline \multicolumn{13}{|c|}{ MS } \\
\hline \multicolumn{13}{|c|}{ First date } \\
\hline S.O.V & $D f$ & $\begin{array}{c}\text { No. of broad } \\
\text { leaved } \\
\text { weeds } \mathrm{m}^{2}\end{array}$ & $\begin{array}{c}\text { Dry } \\
\text { weight } \\
\text { of broad } \\
\text { leaved } \\
\text { weeds } \\
\text { (g) } \mathrm{m}^{2}\end{array}$ & $\begin{array}{l}\text { No. of } \\
\text { narrow } \\
\text { leaved } \\
\text { weeds } \\
\mathrm{m}^{2}\end{array}$ & $\begin{array}{c}\text { dry weight } \\
\text { of narrow } \\
\text { leaved } \\
\text { weeds }(\mathrm{g}) \\
\mathrm{m}^{2}\end{array}$ & $\begin{array}{l}\text { plant } \\
\text { height } \\
(\mathrm{cm})\end{array}$ & $\begin{array}{l}\text { flag } \\
\text { Leaf } \\
\text { area } \\
\mathrm{cm}^{2}\end{array}$ & $\begin{array}{l}\text { Days to } \\
\text { flowering }\end{array}$ & $\begin{array}{l}\text { No. } \\
\text { grain } \\
\text { spike }{ }^{-1}\end{array}$ & $\begin{array}{l}\text { Grain } \\
\text { weight } \\
\text { Spike }^{-1} \\
\text { (g) }\end{array}$ & $\begin{array}{l}1000 \\
\text { grain } \\
\text { weight } \\
\text { (g) }\end{array}$ & $\begin{array}{c}\text { Grain yield } \\
\mathrm{g} \mathrm{m}^{-1}\end{array}$ \\
\hline Block & 2 & 0.188 & 00.52 & 34.56 & 1.84 & $103.02^{*}$ & 16.3 & 0.396 & $8.42^{\star \star}$ & $0.084^{*}$ & $11.7^{*}$ & 143 \\
\hline $\mathrm{H}$ & 3 & $130.85^{\star *}$ & $5.69^{* *}$ & $3972.9^{* *}$ & $3231.5^{\star \star}$ & $191.22^{\star \star}$ & 3.497 & $56.1^{\star *}$ & $303.3^{* *}$ & 0.0175 & $225.5^{\star \star}$ & $\begin{array}{c}31635 \\
* * \\
\end{array}$ \\
\hline $\mathrm{P}$ & 3 & 3.910 & 1.060 & $92.24^{\star *}$ & $62.36^{\star *}$ & 13.34 & 3.795 & $2.47^{\star *}$ & $500.8^{* *}$ & 0.0128 & $175.7^{* \star}$ & $16633^{\star *}$ \\
\hline $\mathrm{HxP}$ & 9 & 6.502 * & 1.482 & $55.37^{\star \star}$ & $56.19^{* \star}$ & 9.41 & 8.362 & $1.42^{* *}$ & $20.1^{\star *}$ & 0.0435 & $38.9^{* *}$ & $3785^{\star *}$ \\
\hline Error & 30 & 2.788 & 1.027 & 15.43 & 3.64 & 21.45 & 6.676 & 0.285 & 1.56 & 0.0209 & 3.275 & 492 \\
\hline Total & 47 & & & & & & & & & & & \\
\hline \multicolumn{13}{|c|}{ Second date } \\
\hline Block & 2 & 0.521 & 0.600 & 2.771 & 5.75 & $2063.8^{\star *}$ & 40.87 & 0.646 & $20.0^{*}$ & $0.0699^{*}$ & 0.957 & 5414 \\
\hline $\mathrm{H}$ & 3 & $68.47^{\star *}$ & $49.10^{\star \star}$ & $161.47^{\star *}$ & $1015.85^{\star *}$ & 68.48 & 3.21 & $36.18^{\star *}$ & $79.3^{\star \star}$ & $0.0592^{*}$ & $19.71^{* *}$ & 3328 \\
\hline $\mathrm{P}$ & 3 & $6.354^{\star \star}$ & $1.842^{\star *}$ & $75.861^{* *}$ & $399.58^{* *}$ & $300.99^{\star *}$ & $53.06^{*}$ & $16.69^{* *}$ & $82.1^{* *}$ & $0.336^{\star *}$ & $108.3^{\star *}$ & $10989^{* *}$ \\
\hline $\mathrm{HxP}$ & 9 & $5.650^{\star *}$ & $2.366^{\star *}$ & $20.213^{* *}$ & $115.45^{\star *}$ & 24.42 & 8.30 & $3.76^{\star *}$ & 10.176 & $0.0527^{*}$ & $17.64^{* *}$ & 1036 \\
\hline Error & 30 & 0.654 & 0.285 & 2.349 & 3.74 & 36.61 & 16.45 & 0.424 & 5.436 & 0.02001 & 2.567 & 2145 \\
\hline Total & 47 & & & & & & & & & & & \\
\hline
\end{tabular}

*, **, indicating significant difference at 0.05 and 0.01 probability levels respectively. $\mathrm{H}-\mathrm{Herbicide,} \mathrm{P}$ phosphorus.

The interaction between herbicides and phosphorus $\left(\mathrm{H}^{*} \mathrm{P}\right)$ caused remarkably differences in most of studied traits, i.e., for number and dry weight of broad and narrow leaved weeds. The check treatment gave the maximum values $(8.8,2.6 \mathrm{~g}, 44.5,37.9 \mathrm{~g}$ and $6.7,5.3 \mathrm{~g}, 10.1 \mathrm{~cm}$ and $22.1 \mathrm{~g}$ in first and second dates while, $\mathrm{H}_{3}$ level exhibited the minimum values $1.8,1.0 \mathrm{~g}, 6.6,3.7$, $1.4,0.8$ and $1.9 \mathrm{~g}$ in both dates(Table 3). Regarding the phosphorus effect, results in (table 4) showed that the phosphorous levels gave worthily effect on the whole traits with except of number and dry weight of broad leaved weeds, plant height, leaf area and grain weight spike ${ }^{-1}$ in first date, and leaf area in second date. The date also showed the effect of four levels of phosphorous in number and dry weight of narrow leaved weeds, the Po level gave the maximum values 21.2 and $16.2 \mathrm{~g}$. For yield and yield components the $\mathrm{P}_{3}$ level produced the highest value $(40.0,32.2 \mathrm{~g}$ and $330.5 \mathrm{~g})$ for number of grain spike $^{-1}$, 1000-grain weight and grain yield, respectively in first date. In second date the Po level was superior in number and dry weight of broad and narrow leaved weeds with values 4.2, $2.9 \mathrm{~g}, 9.2$ and $19.0 \mathrm{~g}$, respectively. For plant height and leaf area the $\mathrm{P}_{2}$ and $\mathrm{P}_{3}$ level produced the highest value, $103.4 \mathrm{~cm}$ and $28.9 \mathrm{~cm}^{2}$. For the earliest days to flowering, $\mathrm{P}_{1}$ level recorded the lowest value 121.9 days. The $\mathrm{P}_{3}$ level was superior in most yield components and gave $1.1 \mathrm{~g}, 2.9 \mathrm{~g}$ and $298.3 \mathrm{~g}$ for grain weight spike ${ }^{-1}, 1000$-grain weight and grain yield. These results indicate that phosphorus has an important effect on the grains in spike because the phosphorus is important in cell division and development of new tissue, phosphorus is also associated with complex energy transformation in the plant and the $\mathrm{H}_{3}$ level was more effective to kill the most broad and narrow leaved weeds compared to the weedy check and caused noticable increasing in grain yield. Similar results regarding herbicide and phosphorous levels were reported by (2) and (24). 
Table (3): Influence of integrate Topic and Granstar herbicides and phosphorous levels on weeds and yield and yield components of bread wheat.

\begin{tabular}{|c|c|c|c|c|c|c|c|c|c|c|c|}
\hline \multicolumn{12}{|c|}{ First date } \\
\hline Treat. & $\begin{array}{c}\text { No of } \\
\text { broad } \\
\text { leaved } \\
\text { weeds } \\
\mathrm{m}^{2}\end{array}$ & $\begin{array}{c}\text { Dry } \\
\text { weight of } \\
\text { broad } \\
\text { leaved } \\
\text { weeds } \\
\text { (g) } \mathrm{m}^{2}\end{array}$ & $\begin{array}{c}\text { No of } \\
\text { narrow } \\
\text { leaved } \\
\text { weeds } \\
\mathrm{m}^{2}\end{array}$ & $\begin{array}{c}\text { dry weight } \\
\text { of narrow } \\
\text { leaved } \\
\text { weeds }(\mathrm{g}) \\
\mathrm{m}^{2}\end{array}$ & $\begin{array}{l}\text { plant } \\
\text { height } \\
(\mathrm{cm})\end{array}$ & $\begin{array}{l}\text { flag Leaf } \\
\text { area } \mathrm{cm}^{2}\end{array}$ & $\begin{array}{l}\text { Days to } \\
\text { flowering }\end{array}$ & $\begin{array}{l}\text { No. } \\
\text { grain.spike } \\
11\end{array}$ & $\begin{array}{c}\text { Grain } \\
\text { weigSpik } \\
\mathrm{e}^{-1}(\mathrm{~g})\end{array}$ & $\begin{array}{c}1000 \\
\text { grain } \\
\text { weight } \\
\text { (g) }\end{array}$ & $\begin{array}{c}\text { Grain yield } \\
\mathrm{g} \mathrm{m}^{2}\end{array}$ \\
\hline $\mathrm{H}_{0}$ & $8.8 \mathrm{a}$ & $2.6 \mathrm{a}$ & $44.5 \mathrm{a}$ & $37.9 a$ & $101.3 a$ & $22.3 \mathrm{a}$ & 141.d & $26.8 \mathrm{c}$ & $0.9 \mathrm{a}$ & $25.3 \mathrm{c}$ & $265.5 b$ \\
\hline $\mathrm{H}_{1}$ & $3.0 \mathrm{~b}$ & $2.1 \mathrm{ab}$ & $6.9 \mathrm{c}$ & $4.8 \mathrm{c}$ & $96.4 \mathrm{ab}$ & $21.5 \mathrm{a}$ & 143.c & $35.1 \mathrm{~b}$ & $0.9 \mathrm{a}$ & $26.8 \mathrm{c}$ & $260.7 \mathrm{~b}$ \\
\hline $\mathrm{H}_{2}$ & $2.1 \mathrm{~b}$ & $1.9 a b$ & $11.7 \mathrm{~b}$ & $7.1 \mathrm{~b}$ & $93.1 \mathrm{~b}$ & $22.4 \mathrm{a}$ & 144.b & $36.7 \mathrm{a}$ & $1.0 \mathrm{a}$ & $31.9 \mathrm{~b}$ & $283.0 \mathrm{~b}$ \\
\hline $\mathrm{H}_{3}$ & $1.8 \mathrm{~b}$ & $1.0 \mathrm{~b}$ & $6.6 \mathrm{c}$ & $3.7 \mathrm{c}$ & $92.5 \mathrm{~b}$ & $21.4 \mathrm{a}$ & 146.a & $37.9 \mathrm{a}$ & $1.0 \mathrm{a}$ & $34.6 \mathrm{a}$ & $370.6 \mathrm{a}$ \\
\hline$P_{0}$ & $3.7 \mathrm{a}$ & $1.7 \mathrm{a}$ & $21.2 \mathrm{a}$ & $16.2 \mathrm{a}$ & $95.7 a$ & $21.1 \mathrm{a}$ & 143.b & $25.0 \mathrm{~d}$ & $0.9 \mathrm{a}$ & $24.3 \mathrm{c}$ & $247.7 \mathrm{c}$ \\
\hline $\mathrm{P}_{1}$ & $3.3 \mathrm{a}$ & $1.9 \mathrm{a}$ & $16.1 \mathrm{~b}$ & $14.2 \mathrm{a}$ & $94.9 a$ & $22.5 \mathrm{a}$ & 143.b & $34.6 \mathrm{c}$ & $0.9 \mathrm{a}$ & $29.3 \mathrm{~b}$ & $283.6 \mathrm{~b}$ \\
\hline $\mathrm{P}_{2}$ & $4.6 \mathrm{a}$ & $2.3 \mathrm{a}$ & 17.8ab & $11.4 \mathrm{~b}$ & $97.4 \mathrm{a}$ & $21.9 \mathrm{a}$ & 143.b & $36.9 \mathrm{~b}$ & $0.9 \mathrm{a}$ & $32.7 \mathrm{a}$ & $318.0 \mathrm{a}$ \\
\hline $\mathrm{P}_{3}$ & $4.2 \mathrm{a}$ & $1.7 \mathrm{a}$ & $14.7 \mathrm{~b}$ & $11.6 \mathrm{~b}$ & $95.4 a$ & $22.0 \mathrm{a}$ & 144.a & $40.0 \mathrm{a}$ & $1.0 \mathrm{a}$ & $32.2 \mathrm{a}$ & $330.5 \mathrm{a}$ \\
\hline \multicolumn{12}{|c|}{ Second date } \\
\hline $\mathrm{H}_{0}$ & $6.7 \mathrm{a}$ & $5.3 \mathrm{a}$ & $10.1 \mathrm{a}$ & $22.1 \mathrm{a}$ & $97.3 a$ & $27.3 a$ & $123.4 \mathrm{a}$ & $27.7 \quad b$ & $0.9 \mathrm{a}$ & $28.2 a$ & $235.3 \mathrm{a}$ \\
\hline $\mathrm{H}_{1}$ & $2.5 \mathrm{~b}$ & $1.8 \mathrm{~b}$ & $7.3 \mathrm{~b}$ & $13.5 \mathrm{~b}$ & 103.a & $28.3 a$ & $123.2 b$ & $32.1 \mathrm{a}$ & $0.9 \mathrm{a}$ & $25.7 b$ & $272.9 \mathrm{a}$ \\
\hline $\mathrm{H}_{2}$ & $2.0 \mathrm{bc}$ & $1.5 \mathrm{~b}$ & $2.2 \mathrm{c}$ & $4.4 \mathrm{C}$ & 100.a & $27.3 a$ & $122.7 \mathrm{~b}$ & $32.6 \mathrm{a}$ & $0.8 \mathrm{a}$ & $25.7 b$ & $248.4 \mathrm{a}$ \\
\hline $\mathrm{H}_{3}$ & $1.4 \mathrm{c}$ & $0.8 \mathrm{c}$ & $3.2 \mathrm{c}$ & $1.9 \mathrm{~d}$ & 100.a & $28.1 \mathrm{a}$ & $119.7 \mathrm{c}$ & $33.5 \mathrm{a}$ & $1.0 \mathrm{a}$ & $25.4 \mathrm{~b}$ & $263.9 \mathrm{a}$ \\
\hline$P_{0}$ & $4.2 \mathrm{a}$ & $2.9 \mathrm{a}$ & $9.2 \mathrm{a}$ & $19.0 \mathrm{a}$ & $92.9 \mathrm{~b}$ & $24.6 b$ & $120.8 d$ & $27.6 \mathrm{~b}$ & $0.7 \mathrm{c}$ & $22.8 \mathrm{~d}$ & $230.7 b$ \\
\hline$P_{1}$ & $2.6 \mathrm{~b}$ & $1.9 \mathrm{~b}$ & $5.5 \mathrm{~b}$ & $6.9 \mathrm{~b}$ & 102.a & $28.8 a$ & $121.9 \mathrm{c}$ & 32.6 a & $0.9 b c$ & $25.0 \mathrm{c}$ & $238.2 \mathrm{~b}$ \\
\hline $\mathrm{P} 2$ & $3.2 \mathrm{~b}$ & $2.4 a b$ & $5.0 \mathrm{~b}$ & $8.6 \mathrm{~b}$ & $103.4 a$ & $28.6 a$ & $122.8 \mathrm{~b}$ & 32.6 a & $1.0 \mathrm{ab}$ & $27.6 \mathrm{~b}$ & 253.3ab \\
\hline P3 & $2.7 \mathrm{~b}$ & $2.2 \mathrm{~b}$ & $3.2 \mathrm{c}$ & $7.3 \mathrm{~b}$ & $103.1 a$ & $28.9 a$ & $123.5 \mathrm{a}$ & $33.2 \mathrm{a}$ & $1.1 \mathrm{a}$ & $29.7 \mathrm{a}$ & 298.3 a \\
\hline
\end{tabular}

Means having the same letters in the same column are not significantly difference at P-value $>0.05$

The data in table 4. recognized the interaction effect between integrate herbicide and phosphorus levels on weeds and wheat yield. The results revealed that the lowest number and dry weight of broad leaved weeds 1.3 and $0.7 \mathrm{~g}$ obtained from $\mathrm{H}_{3} \mathrm{P}_{2}$ level whilst, the lowest values 3.0 and $1.7 \mathrm{~g}$ recorded for number and weight of narrow leaved weeds produced by $\mathrm{H}_{3} \mathrm{P}_{1}$ level in the first date. Moreover, in second date, a minimum number and dry weight of broad and narrow leaved weeds were obtained by $\mathrm{H}_{3} \mathrm{P}_{1}$ and $\mathrm{H}_{3} \mathrm{P}_{2}$ level and values were $1.3,0.3 \mathrm{~g}, 1.3$ and $1.1 \mathrm{~g}$ respectively. The plant height and leaf area exhibited non significant effect by interaction between herbicide and phosphorous levels in both dates and grains weight spike ${ }^{-1}$ in first date. The earliest days to the flowering was recorded in check treatment and values were 140 and 120 days in first and second date respectively. These traits significantly affected by weed and check treatment and exhibited highly competition between wheat crop and both of broad and narrow leaved weeds. Regarding to yield and yield components the results table 5 showed that the highest number of grains spike ${ }^{-1}, 1000$-grain weight and grain yield were realized by $\mathrm{H}_{3} \mathrm{P}_{3}$ and $\mathrm{H}_{1} \mathrm{P}_{1}$ level for number of grain spike ${ }^{-1}$ in first date and recorded 45.4, $42.5 \mathrm{~g}, \quad 451.1 \mathrm{~g}, \quad 35.3,34.0$ and $312.8 \mathrm{~g}$ respectively. The results of herbicide and phosphorus levels were agreed with (14) and (16), However, these results appear that interaction between $(\mathrm{H} \times \mathrm{P})$ was superior in number of grainspike $^{-1}, 1000$-grain weight and grain yield in both dates. 
Table (4): Effect of interaction of integrate herbicides and phosphorous levels on broad and narrow leaved weeds and yield and its components of bread wheat.

\begin{tabular}{|c|c|c|c|c|c|c|c|c|c|c|c|c|}
\hline \multicolumn{13}{|c|}{ First date } \\
\hline $\mathrm{H}$ & $P$ & $\begin{array}{c}\text { No. of } \\
\text { broad } \\
\text { leaved } \\
\text { weeds } \\
\mathrm{m}^{2}\end{array}$ & $\begin{array}{c}\text { Dry } \\
\text { weight } \\
\text { of } \\
\text { broad } \\
\text { leaved } \\
\text { weeds } \\
\text { (g) } \mathrm{m}^{2-1}\end{array}$ & $\begin{array}{c}\text { No. of } \\
\text { narrow } \\
\text { leaved } \\
\text { weeds } \\
\mathrm{m}^{2}\end{array}$ & $\begin{array}{c}\text { dry } \\
\text { weight of } \\
\text { narrow } \\
\text { leaved } \\
\text { weeds }(\mathrm{g}) \\
\mathrm{m}^{2}\end{array}$ & $\begin{array}{l}\text { plant } \\
\text { height } \\
(\mathrm{cm})\end{array}$ & $\begin{array}{c}\text { flag Leaf } \\
\text { area } \\
\left(\mathrm{cm}^{2}\right)\end{array}$ & $\begin{array}{l}\text { Days to } \\
\text { flowering }\end{array}$ & $\begin{array}{c}\text { No. } \\
\text { grain.spik } \\
e^{-1}\end{array}$ & $\begin{array}{l}\text { Grain } \\
\text { weight } \\
\text { Spike }^{-1} \\
\text { (g) }\end{array}$ & $\begin{array}{c}1000 \\
\text { grain } \\
\text { weight }(\mathrm{g})\end{array}$ & $\begin{array}{c}\text { Grain yield } \\
\mathrm{g} \mathrm{m}^{-2}\end{array}$ \\
\hline \multirow[t]{4}{*}{$\mathrm{H}_{0}$} & $\mathrm{P}_{0}$ & $8.7 a b c$ & 2.3ab & $52.0 \mathrm{a}$ & $45.0 \mathrm{a}$ & $100.2 a$ & $20.7 \mathrm{a}$ & $140.7 \mathrm{e}$ & $21.0 \mathrm{~h}$ & $0.9 \mathrm{a}$ & $20.7 \mathrm{e}$ & $231.1 \mathrm{~d}$ \\
\hline & $\mathrm{P}_{1}$ & $5.3 \mathrm{bcd}$ & 1.7ab & 41.3ab & $43.7 \mathrm{a}$ & $101.2 \mathrm{a}$ & $21.9 \mathrm{a}$ & $140.7 \mathrm{e}$ & $24.5 \mathrm{gh}$ & $0.8 \mathrm{a}$ & 22.8de & $269.9 \mathrm{~cd}$ \\
\hline & $\mathrm{P}_{2}$ & $11.7 \mathrm{a}$ & $4.3 \mathrm{a}$ & 44.7ab & $28.4 \mathrm{c}$ & $102.0 \mathrm{a}$ & $25.2 \mathrm{a}$ & 141.3de & $27.7 \mathrm{~g}$ & $0.9 \mathrm{a}$ & $32.3 \mathrm{bc}$ & $314.7 \mathrm{bc}$ \\
\hline & $\mathrm{P}_{3}$ & $9.7 a b$ & 2.3ab & $40.0 \mathrm{~b}$ & $34.5 \mathrm{~b}$ & $101.5 a$ & $21.3 \mathrm{a}$ & 142.0de & $34.1 \mathrm{f}$ & $1.1 \mathrm{a}$ & 25.3de & $246.2 \mathrm{~d}$ \\
\hline \multirow[t]{4}{*}{$\mathrm{H}_{1}$} & $\mathrm{P}_{0}$ & $2.3 \mathrm{~d}$ & $1.7 \mathrm{ab}$ & $3.0 \mathrm{~d}$ & 3.8d-f & $98.3 \mathrm{a}$ & $20.1 \mathrm{a}$ & $144.3 \mathrm{c}$ & $28.0 \mathrm{~g}$ & $0.9 \mathrm{a}$ & 26.0de & $241.8 \mathrm{~d}$ \\
\hline & $\mathrm{P}_{1}$ & $3.3 \mathrm{~d}$ & $2.3 \mathrm{ab}$ & $6.3 \mathrm{~cd}$ & 3.8def & $94.1 \mathrm{a}$ & $21.8 \mathrm{a}$ & $142.3 \mathrm{~d}$ & 35.7 ef & $1.0 \mathrm{a}$ & $26.7 \mathrm{~d}$ & $254.4 \mathrm{~cd}$ \\
\hline & $\mathrm{P}_{2}$ & $2.7 \mathrm{~d}$ & $2.1 \mathrm{ab}$ & $12.7 \mathrm{~cd}$ & $8.1 \mathrm{~d}$ & $97.6 \mathrm{a}$ & $21.3 \mathrm{a}$ & 142.0de & 37.8def & $0.8 \mathrm{a}$ & $27.5 \mathrm{~cd}$ & $265.3 \mathrm{~cd}$ \\
\hline & $\mathrm{P}_{3}$ & $3.7 \mathrm{~cd}$ & 2.2ab & $5.7 \mathrm{~cd}$ & 3.5 def & $95.8 \mathrm{a}$ & $22.7 \mathrm{a}$ & $144.3 \mathrm{c}$ & 38.9 be & $0.9 \mathrm{a}$ & $27.1 \mathrm{~cd}$ & $281.4 \mathrm{bcd}$ \\
\hline \multirow[t]{4}{*}{$\mathrm{H}_{2}$} & $\mathrm{P}_{0}$ & $1.7 \mathrm{~d}$ & $1.6 a b$ & $14.7 \mathrm{~cd}$ & $7.8 \mathrm{de}$ & $94.5 \mathrm{a}$ & $24.5 \mathrm{a}$ & $144.3 \mathrm{c}$ & $25.7 \mathrm{~g}$ & $0.9 \mathrm{a}$ & 24.7de & $237.7 \mathrm{~d}$ \\
\hline & $\mathrm{P}_{1}$ & $2.3 \mathrm{~d}$ & 2.7ab & $13.7 \mathrm{~cd}$ & $7.8 \mathrm{de}$ & $93.0 \mathrm{a}$ & $22.7 \mathrm{a}$ & $144.7 \mathrm{bc}$ & $39.7 \mathrm{bd}$ & $0.8 \mathrm{a}$ & $32.5 b c$ & $270.6 \mathrm{~cd}$ \\
\hline & $\mathrm{P}_{2}$ & $2.7 \mathrm{~d}$ & 2.2ab & $8.7 \mathrm{~cd}$ & 6.5 def & $93.1 \mathrm{a}$ & $20.6 \mathrm{a}$ & $144.3 \mathrm{c}$ & $39.9 \mathrm{bd}$ & $1.1 \mathrm{a}$ & $36.3 \mathrm{~b}$ & $280.5 \mathrm{bcd}$ \\
\hline & $\mathrm{P}_{3}$ & $31.7 d$ & $1.1 \mathrm{~b}$ & $10.0 \mathrm{~cd}$ & $6.5 \mathrm{def}$ & $91.9 \mathrm{a}$ & $21.8 \mathrm{a}$ & $144.3 \mathrm{c}$ & 41.7ac & $1.1 \mathrm{a}$ & $33.9 \mathrm{~b}$ & $343.1 \mathrm{~b}$ \\
\hline \multirow[t]{4}{*}{$\mathrm{H}_{3}$} & $\mathrm{P}_{0}$ & $2.3 \mathrm{~d}$ & $1.3 a b$ & $15.0 \mathrm{c}$ & $8.2 \mathrm{~d}$ & $89.8 \mathrm{a}$ & $21.9 \mathrm{a}$ & 146.0ab & $25.5 \mathrm{~g}$ & $1.0 \mathrm{a}$ & 26.0de & $280.1 \mathrm{bcd}$ \\
\hline & $\mathrm{P}_{1}$ & $2.0 \mathrm{~d}$ & $1.0 \mathrm{~b}$ & $3.0 \mathrm{~d}$ & $1.7 \mathrm{f}$ & $91.4 \mathrm{a}$ & $21.7 \mathrm{a}$ & 146.0ab & $38.5 \mathrm{cde}$ & $1.1 \mathrm{a}$ & $35.4 \mathrm{~b}$ & $339.6 \mathrm{~b}$ \\
\hline & $\mathrm{P}_{2}$ & $1.3 \mathrm{~d}$ & $0.7 \mathrm{~b}$ & $5.0 \mathrm{~cd}$ & 2.6def & $96.7 \mathrm{a}$ & $22.4 \mathrm{a}$ & $146.3 \mathrm{a}$ & 42.3ab & $1.0 \mathrm{a}$ & $34.5 \mathrm{~b}$ & $411.6 \mathrm{a}$ \\
\hline & $\mathrm{P}_{3}$ & $1.7 \mathrm{~d}$ & $1.0 \mathrm{~b}$ & $3.3 \mathrm{~cd}$ & $2.1 \mathrm{ef}$ & $92.3 \mathrm{a}$ & $19.5 \mathrm{a}$ & $147.0 \mathrm{a}$ & $45.4 \mathrm{a}$ & $0.9 \mathrm{a}$ & $42.5 \mathrm{a}$ & $451.1 \mathrm{a}$ \\
\hline \multicolumn{13}{|c|}{ Second date } \\
\hline \multirow[t]{4}{*}{$\mathrm{H}_{0}$} & $\mathrm{P}_{0}$ & $10.0 \mathrm{a}$ & $6.5 \mathrm{a}$ & $13.3 \mathrm{a}$ & $43.4 \mathrm{a}$ & 88.9 & $25.5 \mathrm{a}$ & $120.0 \mathrm{f}$ & $24.4 \mathrm{~d}$ & $0.8 \mathrm{bc}$ & $24.2 \mathrm{dg}$ & $214.2 \mathrm{a}$ \\
\hline & $\mathrm{P}_{1}$ & $4.3 \mathrm{~b}$ & $4.2 \mathrm{~b}$ & $13.3 \mathrm{a}$ & $13.2 \mathrm{~cd}$ & $99.2 \mathrm{a}$ & $29.9 \mathrm{a}$ & $119.3 \mathrm{f}$ & $27.3 \mathrm{~cd}$ & $0.8 \mathrm{bc}$ & $25.8 \mathrm{bf}$ & $232.7 \mathrm{a}$ \\
\hline & $\mathrm{P}_{2}$ & $7.7 \mathrm{a}$ & $6.7 \mathrm{a}$ & $10.3 \mathrm{ab}$ & $17.4 \mathrm{bc}$ & $99.5 \mathrm{a}$ & $27.9 \mathrm{a}$ & $119.3 \mathrm{f}$ & $27.8 \mathrm{bcd}$ & $0.8 \mathrm{bc}$ & $27.1 \mathrm{bf}$ & $243.7 \mathrm{a}$ \\
\hline & $\mathrm{P}_{3}$ & $4.7 \mathrm{~b}$ & $3.9 \mathrm{bc}$ & $3.3 \mathrm{c}-\mathrm{e}$ & $14.3 \mathrm{~cd}$ & 101.a & $26.0 \mathrm{a}$ & 120.0 ef & $31.4 a-d$ & 1.1a-c & $25.7 \mathrm{cf}$ & $250.5 \mathrm{a}$ \\
\hline \multirow[t]{4}{*}{$\mathrm{H}_{1}$} & $\mathrm{P}_{0}$ & $3.0 \mathrm{bc}$ & $1.7 \mathrm{de}$ & $13.3 \mathrm{a}$ & $22.5 \mathrm{~b}$ & $100.6 a$ & $24.4 \mathrm{a}$ & $121.3 \mathrm{de}$ & $25.1 \mathrm{~d}$ & $0.6 \mathrm{c}$ & $23.4 \mathrm{e}-\mathrm{g}$ & $232.2 \mathrm{a}$ \\
\hline & $\mathrm{P}_{1}$ & $2.3 \mathrm{bc}$ & $1.3 \mathrm{de}$ & $4.0 \mathrm{c}-\mathrm{e}$ & $10.9 \mathrm{de}$ & 106.1a & $29.1 \mathrm{a}$ & $123.3 \mathrm{bc}$ & $35.3 \mathrm{a}$ & $0.9 a \_c$ & $23.8-\mathrm{g}$ & $238.0 \mathrm{a}$ \\
\hline & $\mathrm{P}_{2}$ & $1.7 \mathrm{c}$ & $1.6 \mathrm{de}$ & $6.3 \mathrm{~b}-\mathrm{d}$ & $10.4 \mathrm{de}$ & $102.5 a$ & $30.9 \mathrm{a}$ & $123.0 \mathrm{~cd}$ & $34.5 \mathrm{ab}$ & $1.0 \mathrm{ac}$ & $26.9 \mathrm{bf}$ & 272.6 a \\
\hline & $\mathrm{P}_{3}$ & $3.0 \mathrm{bc}$ & $2.5 \mathrm{~cd}$ & $5.7 \mathrm{c}-\mathrm{e}$ & $10.0 \mathrm{de}$ & $103.8 \mathrm{a}$ & $28.7 \mathrm{a}$ & $125.0 \mathrm{ab}$ & $33.7 \mathrm{ac}$ & $1.3 \mathrm{a}$ & $28.7 \mathrm{bcd}$ & $348.7 \mathrm{a}$ \\
\hline \multirow[t]{4}{*}{$\mathrm{H}_{2}$} & $\mathrm{P}_{0}$ & $2.3 \mathrm{bc}$ & $1.9 \mathrm{de}$ & $3.3 \mathrm{c}-\mathrm{e}$ & 6.8 ef & $92.0 \mathrm{a}$ & $24.4 \mathrm{a}$ & $121.3 \mathrm{de}$ & 29.6a-d & $0.7 \mathrm{bc}$ & $23.1 \mathrm{e}-\mathrm{g}$ & $232.8 \mathrm{a}$ \\
\hline & $\mathrm{P}_{1}$ & $2.3 \mathrm{bc}$ & $1.9 \mathrm{de}$ & $1.7 \mathrm{e}$ & $1.4 \mathrm{f}$ & $102.2 a$ & $28.0 \mathrm{a}$ & $122.3 \mathrm{~cd}$ & $33.2 \mathrm{abc}$ & $0.8 a-c$ & $22.7 \mathrm{fg}$ & $237.1 \mathrm{a}$ \\
\hline & $\mathrm{P}_{2}$ & $1.7 \mathrm{C}$ & $0.7 \mathrm{e}$ & $2.0 \mathrm{de}$ & 5.5 ef & $105.2 \mathrm{a}$ & $27.4 \mathrm{a}$ & $123.3 \mathrm{bc}$ & $34.4 \mathrm{ab}$ & $0.8 \mathrm{bc}$ & $25.7 \mathrm{c}-\mathrm{f}$ & $242.5 \mathrm{a}$ \\
\hline & $\mathrm{P}_{3}$ & $1.7 \mathrm{C}$ & $1.7 \mathrm{de}$ & $1.7 \mathrm{e}$ & $4.0 \mathrm{f}$ & $103.2 a$ & $29.2 \mathrm{a}$ & $123.7 a-c$ & 33.3abc & $0.9 a-c$ & $30.1 \mathrm{abc}$ & $281.1 \mathrm{a}$ \\
\hline \multirow[t]{4}{*}{$\mathrm{H}_{3}$} & $\mathrm{P}_{0}$ & $1.3 \mathrm{c}$ & $1.4 \mathrm{de}$ & $6.7 \mathrm{bc}$ & $3.5 \mathrm{f}$ & $89.9 \mathrm{a}$ & $24.0 \mathrm{a}$ & 120.3 ef & $31.3 a-d$ & 0.6 & $20.3 \mathrm{~g}$ & 243.6 a \\
\hline & $\mathrm{P}_{1}$ & $1.3 \mathrm{c}$ & $0.3 \mathrm{e}$ & $3.0 \mathrm{c}-\mathrm{e}$ & $2.0 \mathrm{f}$ & $100.3 \mathrm{a}$ & $28.3 \mathrm{a}$ & $122.7 \mathrm{~cd}$ & $34.5 \mathrm{ab}$ & $0.9 a b c$ & $27.7 \mathrm{~b}-\mathrm{e}$ & $244.8 \mathrm{a}$ \\
\hline & $\mathrm{P}_{2}$ & $1.7 \mathrm{C}$ & $0.6 \mathrm{e}$ & $1.3 \mathrm{e}$ & $1.1 \mathrm{f}$ & $106.2 \mathrm{a}$ & $28.1 \mathrm{a}$ & $125.3 \mathrm{a}$ & $33.8 \mathrm{abc}$ & $1.3 \mathrm{a}$ & $30.6 a b$ & $254.5 \mathrm{a}$ \\
\hline & $\mathrm{P}_{3}$ & $1.3 \mathrm{c}$ & $0.8 \mathrm{e}$ & $2.0 \mathrm{de}$ & $0.9 \mathrm{f}$ & $103.8 \mathrm{a}$ & $31.9 \mathrm{a}$ & $125.3 \mathrm{a}$ & 34.3abc & $1.2 \mathrm{ab}$ & $34.0 \mathrm{a}$ & $312.8 \mathrm{a}$ \\
\hline
\end{tabular}

Means having the same letters in the same column are not significantly difference at $\mathrm{P}$-value $>0$.

Table 5. showed significant effect of integrate herbicide $(\mathrm{H})$ in the whole of studied traits with except of plant height and leaf area, but the phosphorus levels $(\mathrm{P})$ showed highly, remarkable effect in all of traits except dry weight of broad leaved weeds, plant height and leaf area, also the date $(\mathrm{T})$ produced substantially significant effect in all traits except of plant height and grains weight spike ${ }^{-1}$. The interaction between $\mathrm{H}$ x $\mathrm{P}$ exhibited highly significant effect for all studied traits with the exception of the grains weight spike $^{-1}$. the result in the same table indicated that 
an interaction between $\mathrm{H} \times \mathrm{T}$ gave highly significant effect for all traits except of plant height and leaf area whereas, the interaction between $\mathrm{P}$ x $\mathrm{T}$ produced highly significant effect for all traits. However, no significant effect were observed in dry weight of broad leaved weeds, number of narrow leaved weeds, leaf area and grain yield whereas, the interaction between $\mathrm{H} \times \mathrm{P}$ $\mathrm{x} T$ recorded highly significant effect for all studied traits and

non significant for number and dry weight of broad leaved weeds, leaf area and grain yield.

Table (5): Mean square analysis of integrate herbicide and phosphor levels on broad and narrow leaved weeds and yield and its components.

\begin{tabular}{|c|c|c|c|c|c|c|c|c|c|c|c|c|}
\hline \multirow[t]{2}{*}{ S.O.V } & \multirow[t]{2}{*}{ df } & \multicolumn{11}{|c|}{ MS } \\
\hline & & $\begin{array}{c}\text { No. of } \\
\text { broad } \\
\text { leaved } \\
\text { weeds } \\
\mathrm{m}^{2}\end{array}$ & $\begin{array}{c}\text { Dry } \\
\text { weight } \\
\text { of broad } \\
\text { leaved } \\
\text { weeds } \\
\text { (g) } \mathrm{m}^{2}\end{array}$ & $\begin{array}{c}\text { No. of } \\
\text { narrow } \\
\text { leaved } \\
\text { weeds } \\
\mathrm{m}^{2}\end{array}$ & $\begin{array}{c}\text { dry } \\
\text { weight of } \\
\text { narrow } \\
\text { leaved } \\
\text { weeds } \\
\text { (g) } \mathrm{m}^{2}\end{array}$ & $\begin{array}{l}\text { plant } \\
\text { height } \\
(\mathrm{cm})\end{array}$ & $\begin{array}{l}\text { flag } \\
\text { Leaf } \\
\text { area } \\
\mathrm{cm}^{2}\end{array}$ & $\begin{array}{c}\text { Days to } \\
\text { flowering }\end{array}$ & $\begin{array}{c}\text { No. } \\
\text { grain } \\
\text {.spike }\end{array}$ & $\begin{array}{l}\text { Grain } \\
\text { weight } \\
\text { Spike }^{-1} \\
\text { (g) }\end{array}$ & $\begin{array}{l}1000 \\
\text { grain } \\
\text { weight } \\
\text { (g) }\end{array}$ & $\begin{array}{l}\text { Grain } \\
\text { yield } \\
\mathrm{g} \mathrm{m}^{-1}\end{array}$ \\
\hline block & 2 & 0.510 & 1.1316 & 21.95 & 6.41 & $1544.19^{\star \star}$ & $\underset{* *}{33.047}$ & 0.95 & $\underset{* \star}{27.18}$ & $\underset{* *}{0.1494}$ & 7.449 & 3126 \\
\hline $\mathrm{H}$ & 3 & $\underset{* \star}{194.083}$ & $41.554^{*}$ & $\underset{* *}{2659.7}$ & $\underset{*}{3641.94^{*}}$ & 71.63 & 6.995 & $\underset{* *}{84.2}$ & $\underset{* *}{346.06}$ & 0.0456 & $\underset{* *}{168.61}$ & $\underset{* *}{20371}$ \\
\hline$P$ & 3 & $\underset{*}{5.528}$ & 1.2553 & $\underset{* \star}{163.2}$ & $\underset{* \star}{351.66}$ & $\underset{*}{169.29}$ & 7.59 & $\underset{* *}{12.37}$ & $\underset{* \star}{487.74}$ & $0.2372^{* *}$ & $\underset{* \star}{268.33}$ & $\underset{* \star}{25160}$ \\
\hline $\bar{T}$ & 1 & $\underset{* *}{15.042}$ & $\underset{* *}{4.878}$ & $\underset{* *}{3301.76}$ & $\underset{* \star}{202.25}$ & 479.72 & 0 & $11158.6^{\star \star}$ & $\underset{* *}{168.94}$ & 0.0333 & $\underset{* *}{275.98}$ & $\underset{* *}{38079}$ \\
\hline $\mathrm{HxP}$ & 9 & $\underset{* *}{8.852}$ & $\underset{* \star}{3.1405}$ & $\underset{* *}{31.36}$ & $\underset{* \star}{101.21}$ & $\underset{* *}{25.05}$ & $\underset{*}{16.723}$ & $\underset{* *}{2.57}$ & $\underset{* \star}{13.47}$ & 0.0335 & $\underset{* \star}{39.282}$ & 2694 \\
\hline $\mathrm{HxT}$ & 3 & $\underset{*}{5.236}$ & $\underset{* *}{13.2343}$ & $\begin{array}{c}1474.68 \\
* *\end{array}$ & $\underset{* *}{605.42}$ & 188.07 & 0 & $\underset{* *}{8.12}$ & $\underset{* *}{36.49}$ & 0.03107 & $\underset{* *}{76.624}$ & $\underset{* *}{14592}$ \\
\hline PxT & 3 & $\underset{*}{4.736}$ & 1.6475 & 4.9 & $\underset{* *}{110.28}$ & $\underset{*}{145.04}$ & 0 & $\underset{* \star}{6.79}$ & $\underset{* *}{95.13}$ & $\underset{*}{0.11175^{*}}$ & $\underset{* \star}{15.743}$ & 2462 \\
\hline $\begin{array}{c}\mathrm{HxPx} \\
\mathrm{T}\end{array}$ & 9 & 3.301 & 0.7077 & $\begin{array}{c}44.22 \\
\star * \\
\end{array}$ & $\begin{array}{c}70.43 \\
\star * \\
\end{array}$ & $\begin{array}{c}8.78 \\
* \\
\end{array}$ & 0 & $\begin{array}{c}2.61 \\
* *\end{array}$ & $\begin{array}{c}16.8 \\
* *\end{array}$ & $\begin{array}{c}0.06278^{*} \\
*\end{array}$ & $\begin{array}{c}17.234 \\
\star *\end{array}$ & 2128 \\
\hline Error & 62 & 1.672 & 0.6351 & 9.1 & 3.61 & 48.18 & 6.46 & 0.35 & 3.42 & 0.0199 & 2.995 & 1355 \\
\hline Total & 95 & & & & & & & & & & & \\
\hline
\end{tabular}

*, **, indicating significant difference at 0.05 and 0.01 probability level respectively. $\mathrm{H}-$ Herbicide, $\mathrm{P}$ -

Phosphors levels. T- Planting date.

The effect of herbicides levels on weed (broad and narrow leaved weeds) was significant different. The data in table 6 . showed that the highest weed control efficacy $(0.89 \%)$ was recorded by $\mathrm{H} 3$ level, increasing herbicide rates led to significantly increasing weeds control efficiency, while, the $\mathrm{H}_{1}$ level recorded the minimum percent $(0.68 \%)$ of efficiency compared with control. For a number and dry weight of broad and narrow leaved weeds the $\mathrm{P}_{0}$ level produced the maximum values $4.0,2.3 \mathrm{~g}, 15.2$ and $17.6 \mathrm{~g}$, respectively. For plant height and leaf area, these traits were not significantly effects by different levels of herbicides. Also the same traits appeared different effects by phosphorus levels, the maximum plant height was recorded by $\mathrm{P}_{2}$ level with value 100.4 $\mathrm{cm}$ but leaf area appeared non significant effect. The earliest days to flowering the $\mathrm{H}_{0}$ and $\mathrm{P}_{0}$ level gave the minimum days to the flowering and values were 130.4 and 132.3 days because the competition between the weeds and wheat plant was higher in this treatment. Significant differences were also found in yield and yield contributing characters (table 6). The $\mathrm{H}_{3}$ and $\mathrm{P}_{3}$ level produced the highest values $(35.7,1.0 \mathrm{~g}$, $31.4 \mathrm{~g}$ and $317.3 \mathrm{~g}, 36.6,1.0 \mathrm{~g}, 30.9 \mathrm{~g}$ and 314.4 $\mathrm{g}$ ), for number of grains spike ${ }^{-1}$, grain weight, 1000-grain weight, grain yield and the lowest grain yield and yield components were also found in control treatment. The data in the same table confirmed that the first date was superior in the number and dry weight of broad and narrow leaved weeds and also superiority in number of grains spike- ${ }^{1}, 1000$ - grain weight and grain yield and recorded the maximum values (34.1, $29.6 \mathrm{~g}$ and $295.0 \mathrm{~g}$ ) respectively. Similar trends were found by (4) and (24). The results showed that the herbicide reduced the compete between eeds and crops for nutrients, light, causing increase in wheat vigour, tillering, head size and kernel weight. 
Table (6): Influence of integrate herbicides and phosphorous levels on broad and narrow leaved weeds and yield and its components of bread wheat.

\begin{tabular}{|c|c|c|c|c|c|c|c|c|c|c|c|}
\hline $\begin{array}{l}\text { Herbic } \\
\text { ide }\end{array}$ & $\begin{array}{c}\text { No. of } \\
\text { broad } \\
\text { leaf } \\
\text { weeds } \\
\mathrm{m}^{2}\end{array}$ & $\begin{array}{l}\text { dry } \\
\text { weight } \\
\text { of } \\
\text { broad } \\
\text { leaf } \\
\text { weeds( } \\
\text { g) } \mathrm{m}^{2}\end{array}$ & $\begin{array}{c}\text { No. of } \\
\text { narrow } \\
\text { leaf } \\
\text { weeds } \\
\text { m }^{2}\end{array}$ & $\begin{array}{c}\text { dry } \\
\text { weight } \\
\text { of } \\
\text { narrow } \\
\text { leaf } \\
\text { weeds( } \\
\text { g) } \mathrm{m}^{2}\end{array}$ & $\begin{array}{c}\text { plant } \\
\text { height } \\
(\mathrm{cm})\end{array}$ & $\begin{array}{l}\text { Flag Leaf } \\
\text { area } \mathrm{cm}^{2}\end{array}$ & $\begin{array}{c}\text { Days to } \\
\text { flowering }\end{array}$ & $\begin{array}{c}\text { No. } \\
\text { grain.spi } \\
\mathbf{k e}^{-11}\end{array}$ & $\begin{array}{c}\text { Grain } \\
\text { weigSpi } \\
\text { ke }^{-1}(g)\end{array}$ & $\begin{array}{l}1000 \\
\text { grain } \\
\text { weight } \\
\text { (g) }\end{array}$ & $\begin{array}{l}\text { Grain } \\
\text { yield } \\
\mathrm{g} \mathrm{m}^{2}\end{array}$ \\
\hline \multicolumn{12}{|c|}{ Herbicides } \\
\hline $\mathrm{H}_{0}$ & $7.7 \mathrm{a}$ & $4.0 \mathrm{a}$ & $27.3 \mathrm{a}$ & $30.0 \mathrm{a}$ & 99.3 a & $22.3 \mathrm{a}$ & $130.4 \mathrm{c}$ & $27.3 \mathrm{c}$ & $0.9 \mathrm{a}$ & $25.5 \mathrm{c}$ & $250.4 \mathrm{~b}$ \\
\hline $\mathrm{H}_{1}$ & $2.8 \mathrm{~b}$ & $1.9 \mathrm{~b}$ & $7.1 \mathrm{~b}$ & $9.1 \mathrm{~b}$ & 99.8 a & $21.5 \mathrm{a}$ & $133.2 b$ & $33.6 \mathrm{~b}$ & $0.9 \mathrm{a}$ & $26.3 \mathrm{c}$ & $266.8 \mathrm{~b}$ \\
\hline $\mathrm{H}_{2}$ & $2.0 \mathrm{~b} \mathrm{c}$ & $1.7 \mathrm{~b}$ & $7.0 \mathrm{~b}$ & $5.8 \mathrm{c}$ & 96.9 a & $22.4 \mathrm{a}$ & $133.5 \mathrm{~b}$ & $34.7 a b$ & $0.9 \mathrm{a}$ & $28.6 \mathrm{~b}$ & $265.7 b$ \\
\hline $\mathrm{H}_{3}$ & $1.6 \mathrm{c}$ & $0.9 \quad \mathrm{c}$ & $4.9 \mathrm{~b}$ & $2.8 \mathrm{~d}$ & $96.3 \mathrm{a}$ & $21.4 \mathrm{a}$ & $134.9 \mathrm{a}$ & $35.7 \mathrm{a}$ & $1.0 \mathrm{a}$ & $31.4 \mathrm{a}$ & $317.3 \mathrm{a}$ \\
\hline $\mathrm{P}_{0}$ & $4.0 \mathrm{a}$ & $2.3 \mathrm{a}$ & $15.2 \mathrm{a}$ & $17.6 \mathrm{a}$ & $94.3 \mathrm{~b}$ & $21.1 \mathrm{a}$ & $132.3 \mathrm{c}$ & $26.3 \mathrm{c}$ & $0.8 \mathrm{c}$ & $23.6 \mathrm{c}$ & $239.2 \mathrm{c}$ \\
\hline $\mathrm{P}_{1}$ & $2.9 \mathrm{~b}$ & $1.9 \mathrm{a}$ & $10.8 \mathrm{bc}$ & $10.6 \mathrm{~b}$ & $98.5 a b$ & $22.5 \mathrm{a}$ & $132.7 \mathrm{c}$ & $33.6 \mathrm{~b}$ & $0.9 \mathrm{bc}$ & $27.2 \mathrm{~b}$ & $260.9 b c$ \\
\hline $\mathrm{P}_{2}$ & $3.9 \mathrm{ab}$ & $2.3 \mathrm{a}$ & $11.4 b$ & $10.0 \mathrm{~b}$ & $100.4 \mathrm{a}$ & $21.9 \mathrm{a}$ & $133.1 \mathrm{~b}$ & $34.8 \mathrm{~b}$ & $1.0 \mathrm{ab}$ & $30.1 \mathrm{a}$ & $285.7 \mathrm{~b}$ \\
\hline $\mathrm{P}_{3}$ & $3.4 \mathrm{ab}$ & $1.9 \mathrm{a}$ & $9.0 \mathrm{c}$ & $9.5 \mathrm{~b}$ & $99.2 \mathrm{ab}$ & $22.0 \mathrm{a}$ & $134.0 \mathrm{a}$ & $36.6 \mathrm{a}$ & $1.0 \mathrm{a}$ & $30.9 \mathrm{a}$ & $314.4 \mathrm{a}$ \\
\hline $\mathrm{D}_{1}$ & $\mathrm{D}_{1}$ & $3.9 a$ & $1.9 \mathrm{~b}$ & $17.4 \mathrm{a}$ & $13.4 \mathrm{a}$ & $100.3 \mathrm{a}$ & $21.9 \mathrm{a}$ & $143.8 \mathrm{a}$ & $34.1 \mathrm{a}$ & $0.9 \mathrm{a}$ & $29.6 \mathrm{a}$ \\
\hline $\mathrm{D}_{2}$ & $\mathrm{D}_{2}$ & $3.1 b$ & $2.3 \mathrm{a}$ & $5.7 b$ & $10.5 \mathrm{~b}$ & $95.8 \mathrm{~b}$ & $21.9 \mathrm{a}$ & $122.2 \mathrm{~b}$ & $31.5 b$ & $0.9 \mathrm{a}$ & $26.2 \mathrm{~b}$ \\
\hline
\end{tabular}

Numbers fallowed by the same letters in the same column are not significant difference at 0.05 probability level

Table 7. indicated that an interaction between integrate of herbicide and phosphor levels for both dates of planting on wheat and weed yield,. and gave the heights number and dry weight of broad and narrow leaved weeds values 9.3, 32.7 and $44.2 \mathrm{~g}$ recorded by $\mathrm{H}^{0}$ wherever, the lowest values $(1.5,2.7$ and $1.5 \mathrm{~g})$ were recorded by $\mathrm{H}_{3}$ at $\mathrm{P}_{3}$ respectively. Clear decreases found in the above traits as a results of $\mathrm{H}$ and $\mathrm{P}$ application together. According to interaction between $\mathrm{HxP}$ on leaf area and days to flowering, the maximum values of leaf area and days to flowering recorded by $\mathrm{H}_{0} \mathrm{P}_{3}$ which was $25.2 \mathrm{~cm}^{2}$ and 130 days to flowering, while the lowest values of leaf area was $19.5 \mathrm{~cm}^{2}$, and maximum value for days to flowering was 136.2 days.The data in table 8 . showed the highest number of grains spike ${ }^{-1}$, grain weight spike ${ }^{-1}, 1000$ - grain weight and grain yield obtained with $\mathrm{H}_{3} \mathrm{P}_{3}$ level which were $39.9,1.0$ $\mathrm{gm}, 38.3 \mathrm{gm}$ and $381.9 \mathrm{~g}$ respectively. While the lowest values were found in $\mathrm{H}_{0} \mathrm{P}_{0}$ level with the exception grain weight spike ${ }^{-1}$ which was found in $\mathrm{H}_{3} \mathrm{P}_{0}$. These results showed that adding herbicide and phosphorous levels to crops will provide their requirement for a variable phosphorous in balance proportion with nitrogen. The results revealed that all herbicide and phosphorus significantly decreased weed population and direct effected on the wheat yield rate. These results were in agreement with those state out by (20),(19)and

(24)

Table (7): Influence of interaction of integrate herbicides and phosphorus levels .on broad and narrow leaved weeds and yield and its components of bread wheat

\begin{tabular}{|c|c|c|c|c|c|c|c|c|c|c|c|c|}
\hline $\mathbf{H}$ & $\mathbf{P}$ & $\begin{array}{c}\text { No. of } \\
\text { broad } \\
\text { leaved } \\
\text { weeds } \\
\mathrm{m}^{2}\end{array}$ & $\begin{array}{c}\text { Dry } \\
\text { weight } \\
\text { of broad } \\
\text { leaved } \\
\text { weeds } \\
\text { (g) } \mathrm{m}^{2}\end{array}$ & $\begin{array}{c}\text { No. of } \\
\text { narrow } \\
\text { leaved } \\
\text { weeds } \\
\text { m }^{2}\end{array}$ & $\begin{array}{c}\text { dry } \\
\text { weight } \\
\text { of } \\
\text { narrow } \\
\text { leaved } \\
\text { weeds } \\
\text { (g) } \mathrm{m}^{2} \\
\end{array}$ & $\begin{array}{c}\text { plant } \\
\text { height } \\
\text { (cm) }\end{array}$ & $\begin{array}{l}\text { flag Leaf } \\
\text { area } \mathrm{cm}^{2}\end{array}$ & $\begin{array}{l}\text { Days to } \\
\text { flowering }\end{array}$ & $\begin{array}{c}\text { No. } \\
\text { grain.spi } \\
\mathrm{ke}^{-1}\end{array}$ & $\begin{array}{c}\text { Grain } \\
\text { weight } \\
\text { Spike-1 }^{-1} \\
\text { (g) }\end{array}$ & $\begin{array}{c}1000 \\
\text { grain } \\
\text { weight } \\
\text { (g) }\end{array}$ & $\begin{array}{l}\text { Grain } \\
\text { yield } \\
\mathrm{g} \mathrm{m}^{-1}\end{array}$ \\
\hline \multirow[t]{4}{*}{$\mathrm{H}_{0}$} & $\mathrm{P}_{0}$ & $9.3 \mathrm{a}$ & $4.4 \mathrm{ab}$ & $32.7 \mathrm{a}$ & $44.2 \mathrm{a}$ & $94.6 \mathrm{a}$ & $20.7 \mathrm{ab}$ & 130.3 & $22.7 \mathrm{e}$ & $0.8 \mathrm{bc}$ & $22.5 \mathrm{~h}$ & $222.7 \mathrm{e}$ \\
\hline & $\mathrm{P}_{1}$ & $4.8 \mathrm{bc}$ & $2.9 \mathrm{bcd}$ & $27.3 \mathrm{ab}$ & $28.4 \quad b$ & $100.2 \mathrm{a}$ & $21.9 \mathrm{ab}$ & 130.0 & $25.9 \mathrm{de}$ & $0.8 \mathrm{bc}$ & $24.3 f g h$ & 251.3 cde \\
\hline & $\mathrm{P}_{2}$ & $2.0 \mathrm{~d}$ & $5.5 \mathrm{a}$ & $27.5 \mathrm{ab}$ & $22.9 \quad \mathrm{C}$ & $100.8 \mathrm{a}$ & $21.3 \mathrm{ab}$ & $130.3 \mathrm{~g}$ & $27.7 \mathrm{~d}$ & $0.9 \mathrm{abc}$ & $29.7 \mathrm{bcd}$ & 279.2 b-e \\
\hline & $\mathrm{P}_{3}$ & $7.2 \mathrm{ab}$ & $3.1 \mathrm{bc}$ & $21.7 \quad b$ & $24.4 \quad \mathrm{C}$ & $101.7 \mathrm{a}$ & $25.2 \mathrm{a}$ & 131.0 & $32.7 \mathrm{c}$ & $1.1 \mathrm{ab}$ & 25.5 e-h & 248.4 cde \\
\hline
\end{tabular}




\begin{tabular}{|c|c|c|c|c|c|c|c|c|c|c|c|c|}
\hline \multirow[t]{4}{*}{$\mathrm{H}_{1}$} & $P_{0}$ & $2.7 \mathrm{~cd}$ & $1.7 \mathrm{cf}$ & $8.2 \mathrm{cde}$ & $13.1 \mathrm{~d}$ & $99.4 \mathrm{a}$ & $20.1 \mathrm{ab}$ & 132.8 ef & $26.5 \mathrm{~d}$ & $0.8 \quad \mathrm{c}$ & $24.7 \mathrm{eh}$ & $237.0 \mathrm{de}$ \\
\hline & $P_{1}$ & $2.8 \mathrm{~cd}$ & $1.8 \mathrm{c}-\mathrm{f}$ & $5.2 \mathrm{cde}$ & 7.4 ef & $100.1 \mathrm{a}$ & $21.8 \mathrm{ab}$ & 132.8 ef & $35.5 \mathrm{bc}$ & $0.9 \mathrm{abc}$ & $25.2 \mathrm{e}-\mathrm{h}$ & 246.2 cde \\
\hline & $\mathrm{P}_{2}$ & $2.2 \mathrm{~d}$ & $1.8 \mathrm{c}-\mathrm{f}$ & $9.5 \mathrm{c}$ & $9.3 \mathrm{de}$ & $100.1 \mathrm{a}$ & $21.3 \mathrm{ab}$ & $132.5 \mathrm{f}$ & $36.1 \mathrm{abc}$ & $0.9 \mathrm{abc}$ & $27.2 \mathrm{dg}$ & 268.9 b-e \\
\hline & $\mathrm{P}_{3}$ & $3.3 \mathrm{~cd}$ & 2.3 cde & 5.7 cde & 6.7 ef & $99.6 \mathrm{a}$ & $22.7 \mathrm{ab}$ & $134.7 \mathrm{bc}$ & $36.3 a b c$ & $1.1 \mathrm{abc}$ & $27.9 \mathrm{cde}$ & $315.0 \mathrm{abc}$ \\
\hline \multirow[t]{4}{*}{$\mathrm{H}_{2}$} & $\mathrm{P}_{0}$ & $9.7 \mathrm{a}$ & $1.7 \mathrm{c}-\mathrm{f}$ & $9.0 \mathrm{c} \mathrm{d}$ & 7.3 ef & $93.2 \mathrm{a}$ & $21.8 \mathrm{ab}$ & 132.8 ef & $27.6 \mathrm{~d}$ & $0.8 \mathrm{bc}$ & $23.9 \mathrm{~g} \mathrm{~h}$ & $235.2 \mathrm{e}$ \\
\hline & $P_{1}$ & $2.3 \mathrm{~cd}$ & $2.3 \mathrm{c}-\mathrm{f}$ & $7.7 \mathrm{cde}$ & $4.6 \mathrm{fg}$ & $97.6 \mathrm{a}$ & $24.5 \mathrm{ab}$ & $133.5 \mathrm{c}-\mathrm{f}$ & $36.4 \mathrm{abc}$ & $0.8 \mathrm{abc}$ & $27.6 \mathrm{c}-\mathrm{f}$ & $253.9 \mathrm{cde}$ \\
\hline & $\mathrm{P}_{2}$ & $2.2 \mathrm{~d}$ & 1.4 def & $5.3 \mathrm{cde}$ & 6.0 ef & $99.2 \mathrm{a}$ & $22.7 \mathrm{ab}$ & $133.8 \mathrm{cde}$ & $37.1 \mathrm{ab}$ & $0.9 \mathrm{abc}$ & $31.0 \mathrm{~b} \mathrm{c}$ & $261.5 \mathrm{~b}-\mathrm{e}$ \\
\hline & $\mathrm{P}_{3}$ & $1.7 \mathrm{~d}$ & 1.4 def & 5.8 cde & $5.3 \mathrm{fg}$ & $97.6 \mathrm{a}$ & $20.6 \mathrm{ab}$ & 134.0 cde & $37.5 \mathrm{ab}$ & $1.0 \mathrm{abc}$ & $32.0 \mathrm{~b}$ & $312.1 \mathrm{a}-\mathrm{d}$ \\
\hline \multirow[t]{3}{*}{$\mathrm{H}_{3}$} & $\mathrm{P}_{0}$ & $1.8 \mathrm{~d}$ & $1.3 \mathrm{de} f$ & $10.8 \mathrm{c}$ & 5.8 ef & $89.8 \mathrm{a}$ & $21.9 \mathrm{ab}$ & 133.2 def & $28.4 \mathrm{~d}$ & $0.8 \quad \mathrm{c}$ & $23.1 \mathrm{~h}$ & $261.9 \mathrm{~b}-\mathrm{e}$ \\
\hline & $P_{1}$ & $1.7 \mathrm{~d}$ & 0.6 & $3.0 \mathrm{de}$ & $1.8 \mathrm{~g}$ & $95.9 \mathrm{a}$ & $21.7 \mathrm{ab}$ & $134.3 \mathrm{~cd}$ & $36.5 \mathrm{abc}$ & $1.0 \mathrm{abc}$ & $31.5 \mathrm{~b}$ & 292.2 b-e \\
\hline & $\mathrm{P}_{2}$ & $1.5 \mathrm{~d}$ & $0.6 \mathrm{f}$ & $3.2 \mathrm{de}$ & $1.9 \mathrm{~g}$ & $101.5 \mathrm{a}$ & $22.4 \mathrm{ab}$ & $135.8 \mathrm{ab}$ & $38.0 \mathrm{ab}$ & $1.1 \mathrm{a}$ & $32.6 \quad b$ & $333.1 \mathrm{ab}$ \\
\hline
\end{tabular}

Numbers fallowed by the same letters in the same column are not significant difference at 0.05 probability level.

According to data in table 8 , the results showed the interaction of integrate herbicide, phosphorus levels and planting dates on studied traits. The results explained significant effects in all studied traits , the check treatment $\left(\mathrm{H}_{0} \mathrm{D}_{1}\right)$ was superiority in number and dry weight of broad and narrow leaved weeds, and obtained highest values (8.8, $5.3 \mathrm{~g}, 44.5$ and $37.9 \mathrm{~g}$ ) whilst, the $\mathrm{H}_{3} \mathrm{D}_{2}$ recorded the lowest values (1.4, 0.8 and $1.9 \mathrm{~g})$, respectively. For an interaction between phosphorus and planting date, the $P_{2} D_{1}$ gave the maximum value for number of broad leaved weeds whereas, maximum value for dry weight of broad leaved weed obtained by $P_{0} D_{2}$. For number of narrow leaved weed the $P_{0} D_{1}$ gave the high value 21.2 whereas , the maximum value of dry weight (19.0 g) for this trait obtained by $\mathrm{P}_{0} \mathrm{D}_{2}$, this mean the continuous growth of grass throughout different stage of wheat growth of wheat crop. For plant height the $\mathrm{H}_{1} \mathrm{D}_{2}$ treatment gave high average of plant height with value of $103.2 \mathrm{~cm}$. the earliest for days to flowering, the $\mathrm{H}_{0} \mathrm{D}_{2}$ recorded the minimum days(119.7) to flowering. While the first planting date recorded the maximum value (141.2 days) with check treatment of herbicide. The interaction between phosphorus and planting date in the same table showed the maximum plant height was recorded by $\mathrm{P}_{3} \mathrm{D}_{2}$ and minimum days to flowering 122.7 dayes was found in $\mathrm{P}_{2} \mathrm{D}_{2}$. Regarding to data represented in table 9. for yield and yield components the first planting date gave high average number of grains spike ${ }^{-1}$ (37.9), 1000 -grain weight $(34.6 \mathrm{~g})$ and grain yield $370.6 \mathrm{~g}$ with $\mathrm{H}_{3}$ level of herbicide, and same date recorded the high value for the same trait $(40.0,32.2 \mathrm{~g}$ and $330.5 \mathrm{~g}$ ). For number of grains spike ${ }^{-1}, 1000$ - grain weight and grain yield at $\mathrm{P}_{3}$ level. The reasons of superior this traits may be due to the long of first date compared to the second planting date and fast grain filling can reach optimum grain weight and grain filling is the most critical stage, any stress during this stage will negatively affect on 1000grain weight and final seed yield (21). Similar results were also reported by (1) and (17).

Table (8): Influence of interaction of integrate topic and granstar herbicides and phosphorus levels on broad and narrow leaved weeds and yield and its components of bread wheat.

\begin{tabular}{|c|c|c|c|c|c|c|c|c|c|c|c|c|}
\hline $\mathbf{H}$ & D & $\begin{array}{c}\text { No. of } \\
\text { broad } \\
\text { leaved } \\
\text { weeds } \\
\mathbf{m}^{2}\end{array}$ & $\begin{array}{c}\text { Dry } \\
\text { weight } \\
\text { of } \\
\text { broad } \\
\text { leaved } \\
\text { weeds } \\
(\mathrm{g}) \mathrm{m}^{2} \\
\end{array}$ & $\begin{array}{c}\text { No. of } \\
\text { narrow } \\
\text { leaved } \\
\text { weeds } \\
\mathrm{m}^{2}\end{array}$ & $\begin{array}{c}\text { dry } \\
\text { weight } \\
\text { of } \\
\text { narrow } \\
\text { leaved } \\
\text { weeds } \\
\text { (g) } \mathrm{m}^{2} \\
\end{array}$ & $\begin{array}{c}\text { plant } \\
\text { height } \\
(\mathbf{c m})\end{array}$ & $\begin{array}{l}\text { flag } \\
\text { Leaf } \\
\text { area } \\
\mathrm{cm}^{2}\end{array}$ & $\begin{array}{l}\text { Days to } \\
\text { flowering }\end{array}$ & $\begin{array}{l}\text { No. } \\
\text { grain.spi } \\
\mathrm{ke}^{-1}\end{array}$ & $\begin{array}{l}\text { Grain } \\
\text { weight } \\
\text { Spike }^{-1} \\
\text { (g) }\end{array}$ & $\begin{array}{c}1000 \\
\text { grain } \\
\text { weight } \\
\text { (g) }\end{array}$ & $\begin{array}{l}\text { Grain yield } \\
\qquad \mathrm{g} / \mathrm{m}^{-2}\end{array}$ \\
\hline \multirow[t]{2}{*}{$\mathrm{H}_{1}$} & $\mathrm{D}_{1}$ & $3.0 \mathrm{c}$ & $2.1 \mathrm{bc}$ & $6.9 \mathrm{~cd}$ & $4.8 \mathrm{de}$ & $96.4 \mathrm{ab}$ & $21.5 \mathrm{a}$ & $143.2 \mathrm{c}$ & $35.1 \mathrm{~b} \mathrm{c}$ & $0.9 \mathrm{a}$ & $26.8 \mathrm{~cd}$ & $260.7 \mathrm{~b} \mathrm{c}$ \\
\hline & $\mathrm{D}_{2}$ & $2.5 \mathrm{c}$ & $1.8 \mathrm{~b}-\mathrm{e}$ & $7.3 \mathrm{c}$ & $13.5 \mathrm{c}$ & $103.2 \mathrm{a}$ & $21.5 \mathrm{a}$ & $123.2 \mathrm{e}$ & $32.1 \mathrm{~d}$ & $0.9 \mathrm{a}$ & $25.7 \mathrm{~d}$ & $272.9 \mathrm{bc}$ \\
\hline $\mathrm{H}_{2}$ & $\mathrm{D}_{1}$ & $2.1 \mathrm{c}$ & $1.9 \mathrm{bcd}$ & $11.8 \mathrm{~b}$ & $7.1 \mathrm{~d}$ & $93.1 \mathrm{~b}$ & $22.4 \mathrm{a}$ & $144.4 \quad b$ & $36.7 \mathrm{ab}$ & $1.0 \mathrm{a}$ & $31.9 \mathrm{~b}$ & $283.0 \quad b$ \\
\hline
\end{tabular}




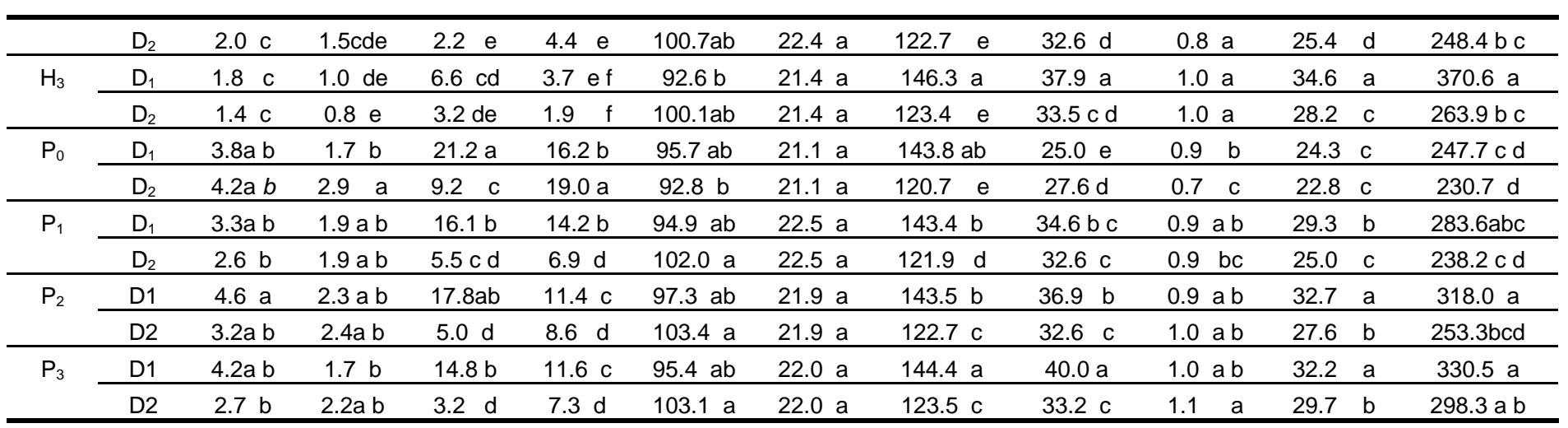

Numbers fallowed by the same letters in the same column are not significant difference at 0.05 probability leve

Results in table 9. exhibited the interaction effect of herbicide, phosphorous levels and planting date. The interaction $\mathrm{H}_{0 \times} \mathrm{P}_{0 \times} \mathrm{D}_{2}$ gave the highest number and dry weight of broad and narrow leaved weeds which, were $(10.0,6.5 \mathrm{~g}$, $43.4 \mathrm{~g}$ ), respectively but, the highest value for number of narrow leaved weeds was 52.0 at $\mathrm{H}_{0} \mathrm{P}_{0}$ $\mathrm{D}_{1}$. The lowest value were 1.3 and 0.8 for number and dry weight of broad leaved weeds The maximum value for number and dry weight of narrow leaved weed obtained by $\mathrm{H}_{3} \mathrm{P}_{2} \mathrm{D}_{2}$ and $\mathrm{H}_{3}$ $\mathrm{P}_{3} \quad \mathrm{D}_{2}$ which were 1.3 and $0.9 \mathrm{~g}$. For days to flowering the earliest days produced by $\mathrm{H}_{0} \mathrm{P}_{1} \mathrm{D}_{1}$ was 119.3 days while, the latest days to flowering recorded by $\mathrm{H}_{3} \quad \mathrm{P}_{3} \quad \mathrm{D}_{1}$ which was 147.0 days because the duratione was 30 days between the first and second date. Data regarding yield and yield components is shown in table 9. Statistical analysis of data that the interaction $\mathrm{H}_{3} \times \mathrm{P}_{3} \times \mathrm{D}_{1}$ showed significant affected on yield and yield component of wheat. Mean values showed that the maximum number of grains spike ${ }^{-1}$ (45.4), 1000grain weight (42.5 g) and grain yield (451.1 g) was found in $\mathrm{H}_{3} \times \mathrm{P}_{3} \times \mathrm{D}_{1}$. while, the minimum values for this traits were 21.0, $20.7 \mathrm{~g}$ and $214.2 \mathrm{~g}$ was found by $\mathrm{H}_{0} \mathrm{P}_{0} \mathrm{D}_{1}$ and $\mathrm{H}_{0} \mathrm{P}_{0} \mathrm{D}_{2}$, respectively .From the results above, all herbicide treatment and phosphor application increased wheat yield and yield components, wheat yield were negatively correlated with weed growth. These results were those of (2) and (11). Who reported that herbicide offer sizeable increase in crop productivity corresponding to their weed control and application of $\mathrm{P}$ under semi arid climate improve the crop growth, yield and its components and seeds quality.

Table (9): Influence of interaction of integrate topic and granstar herbicides and phosphorous levels on broad and narrow leaved weeds and yield and its components of bread wheat.

\begin{tabular}{|c|c|c|c|c|c|c|c|c|c|c|c|c|c|}
\hline $\mathbf{H}$ & $\mathbf{N}$ & D & $\begin{array}{c}\text { NNo, of } \\
\text { broad } \\
\text { leaved } \\
\text { weeds } \\
\mathrm{m}^{2}\end{array}$ & $\begin{array}{c}\text { Dry } \\
\text { weight } \\
\text { of } \\
\text { broad } \\
\text { leaved } \\
\text { weeds } \\
\text { (g) } \mathbf{m}^{2}\end{array}$ & $\begin{array}{c}\text { No of } \\
\text { narrow } \\
\text { leaved } \\
\text { weeds } \\
\mathrm{m}^{2}\end{array}$ & $\begin{array}{c}\text { dry } \\
\text { weight } \\
\text { of } \\
\text { narrow } \\
\text { leaved } \\
\text { weeds } \\
\text { (g) } \mathrm{m}^{2}\end{array}$ & $\begin{array}{c}\text { plant } \\
\text { height } \\
\text { (cm) }\end{array}$ & $\begin{array}{l}\text { flag } \\
\text { leaf } \\
\text { area } \\
\mathrm{cm}^{2}\end{array}$ & $\begin{array}{c}\text { Days to } \\
\text { flowerin } \\
\text { g }\end{array}$ & $\begin{array}{c}\text { No. } \\
\text { grain. } \\
\text { spike }^{-1}\end{array}$ & $\begin{array}{l}\text { Grain } \\
\text { weight } \\
\text { Spike }^{-1} \\
\text { (g) }\end{array}$ & $\begin{array}{c}1000 \\
\text { grain } \\
\text { weight } \\
\text { (g) }\end{array}$ & $\begin{array}{l}\text { Grain } \\
\text { yield } \\
\mathrm{g} \mathrm{m}^{-1}\end{array}$ \\
\hline \multirow[t]{8}{*}{$\mathrm{H}_{0}$} & $\mathrm{P}$ & $\mathrm{D}_{1}$ & $8.7 \mathrm{ab}$ & $2.3 \quad b-e$ & $52.0 \mathrm{a}$ & $45.0 \mathrm{a}$ & $100.2 \mathrm{a}$ & $20.7 \mathrm{a}$ & $140.7 \mathrm{~d}$ & $21.0 \mathrm{n}$ & 0.9 a-e & $20.7 \mathrm{j} \mathrm{k}$ & $231.1 \mathrm{c} \mathrm{d}$ \\
\hline & & $\mathrm{D}_{2}$ & $10.0 \mathrm{a}$ & $6.5 \mathrm{a}$ & 13.3cde & $43.4 \mathrm{a}$ & 88.9 a & $20.7 \mathrm{a}$ & $120.0 \mathrm{ij}$ & $24.4 \mathrm{mn}$ & $0.8 \mathrm{cde}$ & $24.2 \mathrm{~h}-\mathrm{k}$ & $214.2 \quad d$ \\
\hline & $\mathrm{P}$ & $\mathrm{D}_{1}$ & $5.3 \mathrm{~b} \mathrm{c} \mathrm{d}$ & $1.7 \quad b-e$ & $41.3 \quad b$ & $43.7 \mathrm{a}$ & $101.2 \mathrm{a}$ & $21.9 \mathrm{a}$ & $140.7 \mathrm{~d}$ & $24.5 \mathrm{mn}$ & 0.8 a-e & $22.8 \mathrm{i} \mathrm{jk}$ & $269.9 \mathrm{c} \mathrm{d}$ \\
\hline & 1 & $\mathrm{D}_{2}$ & $4.3 \mathrm{c} \mathrm{d}$ & $4.2 \mathrm{abc}$ & 13.3cde & $13.2 \mathrm{efg}$ & $99.2 \quad \mathrm{a}$ & $21.9 \mathrm{a}$ & $119.3 \mathrm{j}$ & $27.3 \mathrm{klm}$ & $0.8 \mathrm{~b}-\mathrm{e}$ & $25.8 \mathrm{f}-\mathrm{k}$ & $232.7 \mathrm{c} \mathrm{d}$ \\
\hline & $P$ & $\mathrm{D}_{1}$ & $11.7 \mathrm{a}$ & $4.3 \mathrm{ab}$ & $44.7 \mathrm{ab}$ & $28.4 \mathrm{~b} \mathrm{c}$ & $102.0 \mathrm{a}$ & $21.3 \mathrm{a}$ & $141.3 \mathrm{~d}$ & $27.7 \mathrm{j}-\mathrm{m}$ & 0.9 a-e & $32.3 \mathrm{~b}-\mathrm{e}$ & $314.7 \mathrm{bcd}$ \\
\hline & 2 & $\mathrm{D}_{2}$ & $7.7 \mathrm{abc}$ & $6.7 \mathrm{a}$ & $10.3 \mathrm{c}-\mathrm{g}$ & $17.4 \mathrm{~d} \mathrm{e}$ & $99.5 \mathrm{a}$ & $21.3 \mathrm{a}$ & $119.3 \mathrm{j}$ & $27.8 \mathrm{i}-\mathrm{m}$ & 0.8 a-e & $27.1 \mathrm{e}-\mathrm{i}$ & $243.7 \mathrm{c} \mathrm{d}$ \\
\hline & $\mathrm{P}$ & $\mathrm{D}_{1}$ & $9.7 \mathrm{a}$ & $2.3 \quad b-e$ & $40.0 \quad b$ & $34.5 \mathrm{~b}$ & $101.5 \mathrm{a}$ & $25.2 \mathrm{a}$ & $142.0 \mathrm{~d}$ & $34.1 \mathrm{~d}-\mathrm{h}$ & $1.1 \mathrm{abc}$ & $25.3 \mathrm{f}-\mathrm{k}$ & $246.2 \mathrm{c} \mathrm{d}$ \\
\hline & 3 & $\mathrm{D}_{2}$ & $4.7 \mathrm{bcd}$ & $3.9 \mathrm{bcd}$ & $3.3 \mathrm{fg}$ & $14.3 \mathrm{ef}$ & $101.8 \mathrm{a}$ & $25.2 \mathrm{a}$ & $120.0 \mathrm{ij}$ & $31.4 \mathrm{~g}-\mathrm{I}$ & $1.1 \mathrm{a}-\mathrm{e}$ & $25.7 \mathrm{f}-\mathrm{k}$ & $250.5 \mathrm{~cd}$ \\
\hline \multirow[t]{3}{*}{$\mathrm{H}_{1}$} & $\mathrm{P}$ & $\mathrm{D}_{1}$ & 2.3 & 1.7 b-e & $3.0 \mathrm{fg}$ & $22.5 \mathrm{c} \mathrm{d}$ & $98.3 \mathrm{a}$ & $20.1 \mathrm{a}$ & $144.3 \mathrm{c}$ & $28.0 \mathrm{i}-\mathrm{m}$ & 0.9 a-e & $26.0 \mathrm{f}-\mathrm{j}$ & $241.8 \mathrm{c} \mathrm{d}$ \\
\hline & 0 & $\mathrm{D}_{2}$ & 3.0 & $1.7 \mathrm{cde}$ & 13.3cde & $3.8 \mathrm{j}-\mathrm{m}$ & $100.6 \mathrm{a}$ & $20.1 \mathrm{a}$ & $121.3 \mathrm{~h} \mathrm{i}$ & $25.1 \mathrm{~m} \mathrm{n}$ & $0.6 \mathrm{de}$ & $23.4 \mathrm{~h}-\mathrm{k}$ & $232.2 \mathrm{c} \mathrm{d}$ \\
\hline & $\mathrm{P}$ & $\mathrm{D}_{1}$ & $3.3 \mathrm{~d}$ & $2.3 \mathrm{~b}-\mathrm{e}$ & $6.3 \mathrm{c}-\mathrm{g}$ & 10.9fgh & $94.1 \quad \mathrm{a}$ & $21.8 \mathrm{a}$ & $142.3 \mathrm{~d}$ & $35.7 \mathrm{c}-\mathrm{g}$ & 1.0 a-e & $26.7 \mathrm{f}-\mathrm{i}$ & $254.4 \mathrm{c} \mathrm{d}$ \\
\hline
\end{tabular}




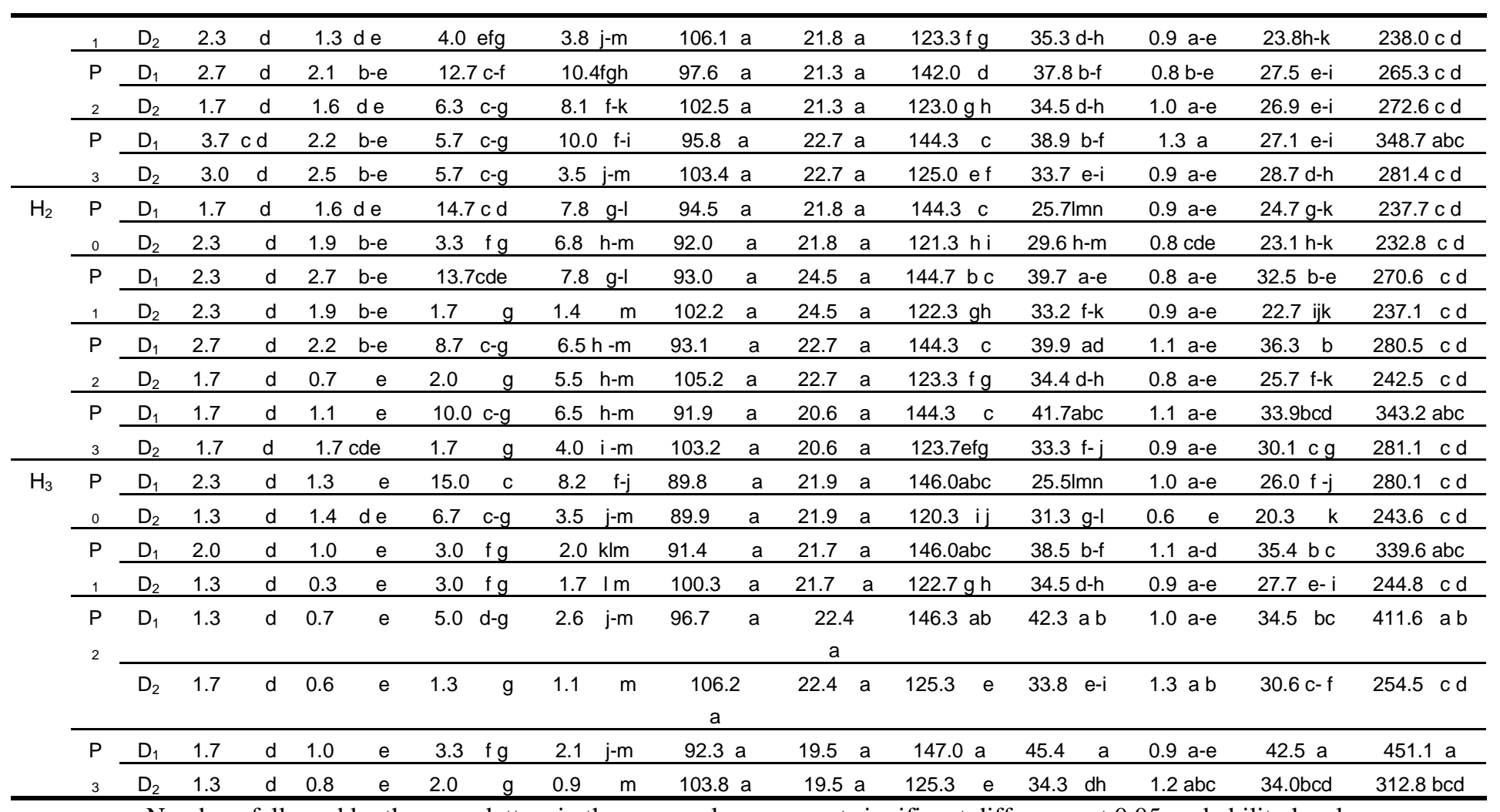

Numbers fallowed by the same letters in the same column are not significant difference at 0.05 probability level.

\section{CONCLUSION}

We conclude that the most effective herbicide treatment $(\mathrm{H} 3)$ and $(\mathrm{P} 3)$ level which provided the maximum reduction in total weed. and because of the environmental concerns, application of the high herbicide dose with high phosphorus level are recommended for improving weed control and high wheat yield and good wheat seed quality.

\section{REFERENCES}

- Ahmed, N . and M. Rashid . 2003. Fertilizer and their use in Pakistan. National fertilizer Development Center. Government of Pakistan : 41-45.

- Ahmed. S. N. E. Jan, Khan R. . Faridullah and N. Din. 2010. Wheat response to phosphorous under climate condition of Juglote, Pakistan. Shrhat J. Agric., 26(2) : 229- 233.

- Ali Y. N, MojtabaAlavi F2, A. A.3.2013. Grarstar and Topic herbicides and split of nitrogen Fertilizer on Controlling weeds of D79-15 wheat conducted in Behbahancity in tarnation Journal of Agriculture and crop seience. 5, (21): 25842587.

- Ashrafi, L. Y. 2009. Study of integrate methods chemical and cultural control of weed to wheat
(Triticutum estivum L.). Journal of Agriculture Science, 1(2): 1 - 12.

- Awaad, M. S., A. A. Rashed and M. A Bayoumi M. A. 2009. Effect of farmyard manare combined with some phosphate source on the productivity of canola plant grown on a sandy soil. Research Journal of Agriculture and Biological Sciences, 5: 1176-1181.

- Barber. S.1995. Soil Nutrient Bioavailability. John Wiley and Sons Inc USA.

- Barwary. S. S. 2012. Iron efficient and inefficient Wheat Genotypes Growth in Iron - Deficient calcareous soil, M. Sc Thesis, Collage of Agriculture, Duhok University, Republic of Iraq pp: 93-129.

- Bib. B, H. Gul and Noor K.M. 2008. Effect f herbicides and wheat population on control of weed in wheat . Pakistan J. of Weed Sci.Res.14: 111-119.

- Cheema.M.S and M.Akhtar., 2005. Efficacy of different post emergence herbicides and their environmen Pak. J. Weed Sci. Res. 11(1-2):2329.

- El-Kholy,R. M. A, W. I. Abouamer and M. M. Ayoub. 2013. Efficacy of some herbicides for controlling broad-leaved weeds in wheat fields. 
Journal of Applied Sciences Research, 9(1): 945-951, 2013.

- Farzad. S and S. Saeed 2015. Efficacy evaluation of some herbicides and different nitrogen for weed management and yield attibutesn weed. WALLA Journal. 3: 39 -43.

- Garkova, A. N.,M. M. Rusyaeve, O. V. Nushtaeve, Y. N. Aroslan Kinq and Lukatin. A. S. 2011. Treatment with the herbicide Granstar induces oxidative stress in cereal leaves. Russin J. of plant physiology 58, 1047-1081.

- Kacar. B,and A. V. Katkat. 2011. Bitiki besleme. Nobel Yayinlari( S. Baski): 1-678 Turkey.

- Kaleem, S. Ansar. M. A. Sher, A. Ahed, G and M. Reashid. 2009. Effect of phosphorous on yield and yield component of wheat variety in alab-91 under rain fed conditions. Sarhad. J. Agric., 25 (1): 21-24.

- Khalil MF, Hassan G, Ahmad G and Shah NH. Individual and combined effect of different herbicides on weed control in wheat. Pakistan Journal of Weed Science Research 2008; 14(34): 131-139.

- Khaliq, A., A. Matloop, Tanveer, A. Ahsan. A. Areeb F. and Abbas. N. 2011 reduced doses pf sulfonylurea herbicide for weed management in wheat fields of Punjab. Pakistan. Chilean J. Agriculture Research, 71 (3): 22- 27.

- Kumar, V., R. S Samarsingh; Chhokar; R. K; D. C. Malik, Brainard and LadHa. J, K, 2013. Weed management strategies to reduce herbicide use in Iero- till rice-wheat cropping system of indo gangetic plains. Weed Technology. 27: 24-254.

- Mata, A. E., and S. C. Brown. 1989. Effect of level and method of phosphor placement on productivity of Durum- wheat in a
Mediterranean Environment. Crop yield and $\mathrm{p}$ Uptake. Fertilizer Research. 20. 75-82.

- Marwat . k. B; S. Muhamme d, H. Zahid; B. Gul and Rashid. H. 2008. Study of various weed management practices for weed control in wheat under irrigated conditions. Pak. J. Weed Sci. Res., 14 (1-2): 1-8.

- Mosali J., K. Desta, R. K. Teal et al. 2006.“ Effect of foliar application of phosphorus on winter wheat grain yield, phosphorus uptake, and use efficiency," Journal of Plant Nutrition, 29,( 12): 2147-2163.

- Riaz M, Azim Malik M, MahmoodTZ, Jamil M (2006) Effect of Various Weed Control Methods on Yield and Yield Components of Wheat under Different Cropping Patterns. International Journal of Agriculture \& Biology8: 636-640.

- Robertson LD and G Lowry. 2004. Seed Quality and Seed Production. p. 19-21.

- Shad. R.A 1987.Status of Weed Science Activities in Pakistan. Progressive Farming, 7(1) , pp :10-16.

- Shahzad,M. A; M, A.Nadeem, M. A. Sarwar, Ud. Bin. Naseer and Eahi F. 2012. Compar -ative efficacy of different -post emergence herbicides in wheat ( Triticum estivum ), Pak. J. Agr. Sci., 49 (1): 27-34.

- Staats, M., Cuenca, A., Richardson, J.E.,

Vrielink-van Ginkel, R., Petersen, G., Seberg, O. \& Bakker, F.T. 2011. DN damage in plant herbarium tissue. PLoS ONE 6(12): e28448.http://dx.doi.org/10.1371/journal.pone.0 028448

- Tesfay amare. S. and Kassahun Z. 2014. Effect of weed management methods on weeds and wheat (Triticum aestivum L.) yield. African Journal of Agricultural Research. 9 : 1914-1920. 


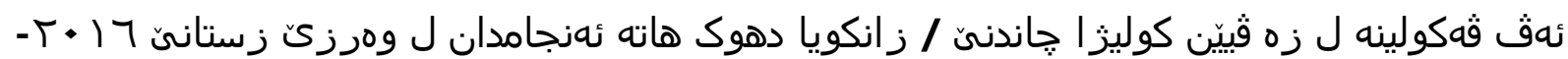

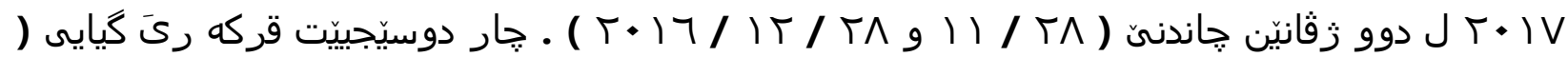

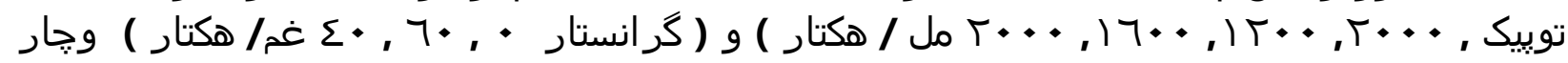

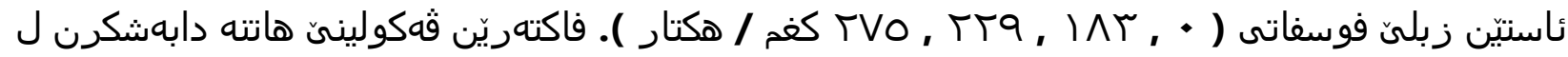

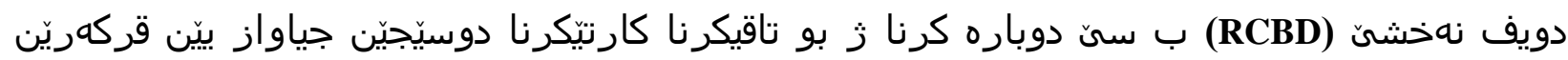

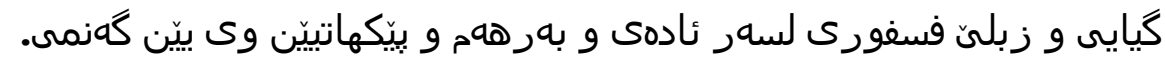

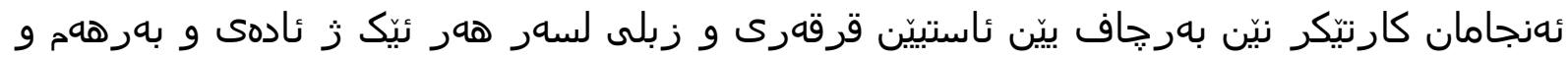

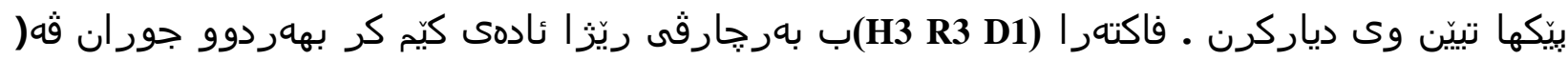

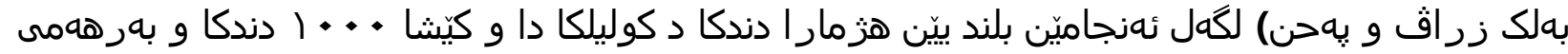
توثن كو ( 45.4 و 42.5 غم و

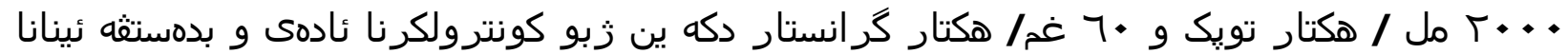

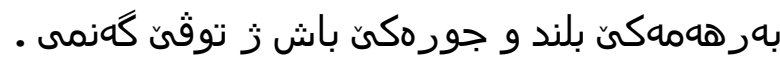

حنطة الخبز وتأثيراتها باظافة مستويات من مخلوط مبيدي الادغال ( توبك + كرانستار) والسماد

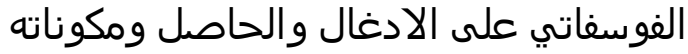

نفذت تجربة حقلية في حقل كلية الزراعة جامعة دهوك خلال موسم الشتوي 2016- 2017 وبموعدين

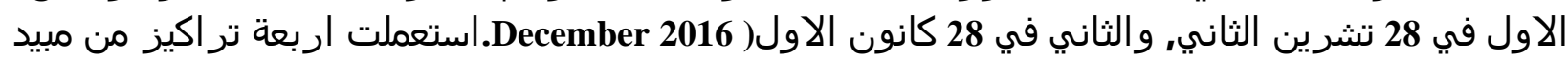

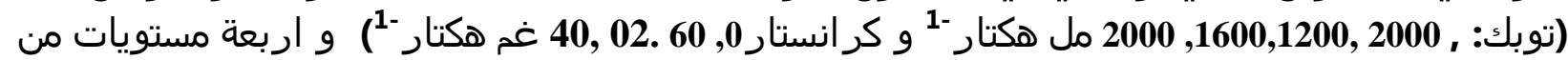

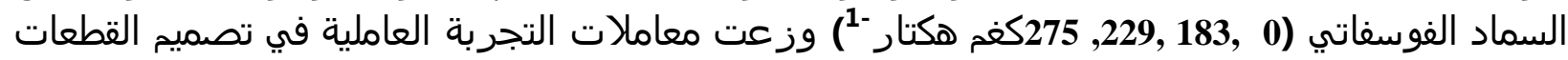

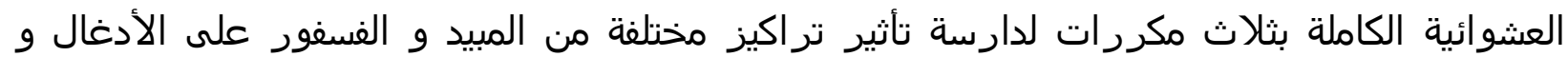

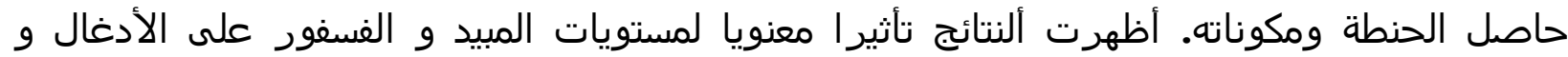

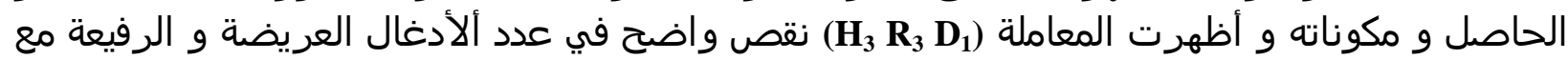

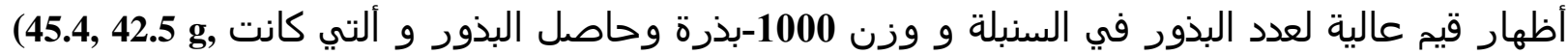
(Topic:2000 ml. ha-1 and Granstar,60 gm ha-1)275 kg ha ha $^{-1}$.and 451.1 g) للسيطرة على نمو ألأدغال و حاصل وهي باسنعمال عالي و نوعية جيدة من بذور الحنطة. 